\title{
Generation of Tunable THz Pulses
}

\author{
J. Degert, S. Vidal, M. Tondusson, C. D’Amico, J. Oberlé and É. Freysz
}

Additional information is available at the end of the chapter

http://dx.doi.org/10.5772/46234

\section{Introduction}

Many phenomena in materials science, physics, chemistry, biology and medicine involve fundamental processes with a spectral signature in the $\mathrm{THz}$ range [20]. However, for many years, these processes have been little studied due to the lack of high quality $\mathrm{THz}$ sources. Fortunately, the recent developments in $\mathrm{THz}$ technology have filled this so-called $\mathrm{THz}$ gap [28]. With the emergence of reliable $\mathrm{THz}$ sources, the need for arbitrarily shaped $\mathrm{THz}$ pulses dedicated to specific applications like communications, signal processing, spectroscopy or coherent/optimal control is more and more felt. Concerning coherent control, selective excitations in the $\mathrm{THz}$ spectral region of phonon modes in molecular crystal [41], charge oscillations in semiconductor heterostructures $[5,26]$ or phonon-polaritons in ferroelectric crystal $[9,40]$ have already been achieved by means of temporally shaped near-infrared laser pulses. From this point of view, the possibilities of control would be increased if one makes it possible to excite directly the system with shaped $\mathrm{THz}$ pulses [27]. To this end, several methods have been developed to extend the generation of arbitrary pulse shapes from the visible and mid-infrared spectral region up to the THz range [1, 8, 10, 16-19, 21, 30, 31, 43]. All are based on the generation of $\mathrm{THz}$ pulses by excitation of different types of emitters with spatially and/or temporally shaped optical pulses.

Here, we will focus our attention on the generation, tuning and shaping of narrow-band $\mathrm{THz}$ pulses. Such pulses have already been implemented by means of various pulse shaping techniques which differ from each other by their respective spectral width, their ease of use and their versatility. Some of them use traditional pulse shaping setup: for example, Sohn et al. [30] used a specially designed mask placed in the Fourier plane of a zero-dispersion line to produce modulated optical pulses subsequently photomixed to generate $\mathrm{THz}$ pulses tunable from 0.5 to $3 \mathrm{THz}$, with a spectral width of $\sim 500 \mathrm{GHz}$. Despite its tunability, this method is limited by the use of a non programmable mask. Likewise, using optical pulse shaping with a liquid crystal spatial light Fourier filter combined with optical rectification in ZnTe, Ahn et al. were able to generate $\mathrm{THz}$ waves tunable from 0.5 to $2 \mathrm{THz}$, with a spectral bandwidth of $\sim 200 \mathrm{GHz}$ [1]. To do this, they have implemented a Gerchberg-Saxton algorithm to find the best spectral phase required for the optical pulse in order to generate the desired $\mathrm{THz}$ pulse. However, this technique, characterized by its great ability to generate various pulse 
shapes, has one drawback: it relies on the ability of the algorithm to converge towards the expected solution. On the other hand, interesting examples of tunable narrow-band $\mathrm{THz}$ pulses with possibilities of shaping the waveform have also been reported in lithium niobate crystals. For example, Lee et al. [16, 18, 19] employed a single pulse or a pair of temporally separated optical pulses rectified in PPLN crystals with a specially engineered domain structure, generating very narrow $\mathrm{THz}$ pulses (bandwidth $\sim 25 \mathrm{GHz}$ ) tunable between 0.5 and $2.5 \mathrm{THz}$. Nevertheless, it requires a new crystal with a specific domain structure for each temporal shape or an adjustment of the crystal position to tune the frequency. At last, using a transient polarization grating induced by two femtosecond laser pulses propagating in a $\mathrm{LiNbO}_{3}$ crystal, Stepanov et al. [31] were able to generate and to shape $\mathrm{THz}$ pulses tunable from 0.5 to $3 \mathrm{THz}$ with a bandwidth of $100 \mathrm{GHz}$. In this experiment, the tunability is controlled by the angle between the pump beams and the shaping is accomplished by filtering with a slit or a shield the spatial intensity distribution of the pump beams. While the spatial filtering can be done with a liquid crystal modulator, enabling thus to do optimal control experiments, the control of the tunability is less flexible.

In the present chapter, we present two different techniques that make it possible to generate tunable $\mathrm{THz}$ pulses. The first one, that can be regarded as analytical, is based on the spectral tailoring of an ultrashort femtosecond laser pulse, whereas the second one is relying on the spatial shaping of the transverse profile of the femtosecond laser beam. In both techniques, liquid crystal devices are used to modulate in the spatial or spectral domain the femtosecond pulses that are incident on a nonlinear crystal in which optical rectification is taking place. Hence, it is flexible and needs no moving mechanical parts or Mach-Zender like interferometers. We demonstrate that the first one makes it possible to generate $\mathrm{THz}$ pulses tunable between 0.5 and $2.5 \mathrm{THz}$ with spectral bandwidth as narrow as $140 \mathrm{GHz}$. The second one, based on a geometrically-assisted optical rectification technique, enables us to generate $\mathrm{THz}$ spatiotemporal interferences in the intermediate field zone beyond the rectifying crystal. We will show that the spatiotemporal properties of the generated $\mathrm{THz}$ field in the intermediate zone are tightly tied to the geometry of the transverse profile of the laser beam attaining the rectifying crystal. Therefore by shaping the transverse beam profile one can change the temporal and the transverse profile of the emitted $\mathrm{THz}$ pulse.

\section{Model and preliminary analysis of $\mathrm{THz}$ generation by optical rectification in zinc blende crystals}

Whatever the way used to obtain shaped $\mathrm{THz}$ waves, it is strongly correlated to the physical mechanisms involved in the $\mathrm{THz}$ wave generation. Here, since our attention will be focused on $\mathrm{THz}$ generation by optical rectification in Zinc Blende crystals, we are going to remind, at first, the main theoretical results concerning this process. Then, based upon these latter, we will show how one can obtain shaped $\mathrm{THz}$ pulses.

Let us consider an ultrashort near-infrared (NIR) laser pulse exciting, at normal incidence, a ZnTe emitter cut along the $\langle 110\rangle$ plane, with a thickness $L_{e}$. Assuming a propagation in the $z$-direction, we can write its electric field in the following form:

$$
E(t, x, y, z)=u(x, y, z) \mathcal{E}(t) \exp \left[i\left(\omega_{0} t-k_{0} z\right)\right],
$$

where $u(x, y, z)$ and $\mathcal{E}(t)$ are, respectively, the spatial and temporal amplitudes of the laser beam, $\omega_{0}$ its carrier frequency (corresponding to a central wavelength $\lambda_{0}=2 \pi c / \omega_{0}, c$ is 
the velocity of light in vaccum), and $k_{0}$ the mean wave vector $\left(k_{0}=\omega_{0} n\left(\omega_{0}\right) / c\right.$, where $n$ is the refractive index of $\mathrm{ZnTe}$ ). Here, we assume that this pulse is not modified during its propagation in the nonlinear crystal, so that:

$$
u(x, y, z)=\mathcal{F}(x, y) \exp \left(i \frac{\omega z}{v_{g}}\right)
$$

$v_{g}$ being the group velocity of the laser pulse: $v_{g}=c / n_{g}\left(\omega_{0}\right)$, where $n_{g}\left(\omega_{0}\right)=n\left(\omega_{0}\right)+$ $\omega_{0}(d n / d \omega)_{\omega_{0}}$ is the group index of the NIR pulse.

At each point of the medium, this pulse induces via the frequency difference between its different spectral components a second order nonlinear polarization whose spectral components $\Omega$ lie in the terahertz range. More precisely, the nonlinear polarization induced at frequency $\Omega$ is given by:

$$
P^{(2)}(\Omega, x, y, z)=\varepsilon_{0} \chi^{(2)}(\Omega) \int \frac{d \omega}{2 \pi} E(\omega, x, y, z) E^{*}(\omega-\Omega, x, y, z),
$$

where $\varepsilon_{0}$ is the vacuum permittivity, $\chi^{(2)}$ the second order nonlinear susceptibility of ZnTe, and $E(\omega, x, y, z)=\int E(t, x, y, z) \exp (i \omega t) d t$. Note that, in Eq. (3), we have assumed that the spectral dependence of $\chi^{(2)}$ in the NIR range is negligible. Taking into account Eqs. (1) and (2), and introducing the spectral amplitude of the optical field $\mathcal{E}(\omega)=A(\omega) \exp [i \phi(\omega)]=$ $\int \mathcal{E}(t) \exp (i \omega t) d t$, one obtains:

$$
P^{(2)}(\Omega, x, y, z)=\varepsilon_{0}|\mathcal{F}(x, y)|^{2} e^{i \frac{\Omega z}{v g}} \chi^{(2)}(\Omega) C(\Omega),
$$

where $C(\Omega)$ is the power spectrum of the rectified NIR pulse, given by:

$$
\begin{aligned}
C(\Omega) & =\int|\mathcal{E}(t)|^{2} \exp (i \Omega t) d t, \\
& =\int \frac{d \omega}{2 \pi} \mathcal{E}(\omega) \mathcal{E}^{*}(\omega-\Omega), \\
& =\int \frac{d \omega}{2 \pi} A(\omega) A(\omega-\Omega) e^{i[\phi(\omega)-\phi(\omega-\Omega)]} .
\end{aligned}
$$

From a quantum mechanical point of view, $C(\Omega)$ is proportional to the probability amplitude that two photons of the NIR pulse with frequencies $\omega$ and $\omega-\Omega$ interact in the nonlinear crystal to generate a THz photon at frequency $\Omega$.

So, this second order polarization emits $\mathrm{THz}$ radiations at different frequencies $\Omega$, and, consequently, acts as a source term in the wave equation for the $\mathrm{THz}$ wave generated by optical rectification. This latter equation, written in the spatial and spectral Fourier domains, is given by $[2,6]$

$$
\left[\frac{\partial^{2}}{\partial z^{2}}+k_{\|}^{2}\right] E_{\mathrm{THz}}\left(\Omega, k_{x}, k_{y}, z\right)=-\frac{\Omega^{2}}{c^{2}} \chi^{(2)}(\Omega) C(\Omega) \mathcal{G}\left(k_{x}, k_{y}\right) e^{i \Omega z / v_{g}},
$$

where

$$
\mathcal{G}\left(k_{x}, k_{y}\right)=\frac{1}{(2 \pi)^{2}} \iint|\mathcal{F}(x, y)|^{2} e^{-i\left(k_{x} x+k_{y} y\right)} d x d y,
$$


and $k_{\|}=\sqrt{k^{2}(\Omega)-k_{\perp}^{2}}$, with $k_{\perp}=\sqrt{k_{x}^{2}+k_{y}^{2}}$ and $k(\Omega)=n(\Omega) \Omega / c$ (wave vector of the $\mathrm{THz}$ wave).

At the exit of the ZnTe crystal $\left(z=L_{e}\right)$, the amplitude of each spectral component of the $\mathrm{THz}$ pulse generated by optical rectification is given by $[6,7]$

$$
\begin{aligned}
E_{\mathrm{THz}}\left(\Omega, k_{x}, k_{y}, L_{e}\right)=E_{\mathrm{THz}}\left(\Omega, k_{\perp}, L_{e}\right) & =i L_{e} \frac{\Omega^{2}}{c^{2}} \frac{\chi^{(2)}(\Omega)}{k_{\|}(\Omega)+\Omega / v_{g}} e^{i\left[k_{\|}(\Omega)+\Omega / v_{g}\right] \frac{L_{e}}{2}} \\
& \times C(\Omega) \mathcal{G}\left(k_{\perp}\right) \operatorname{sinc}\left[\frac{L_{e} \Delta k}{2}\right],
\end{aligned}
$$

where $\mathcal{G}\left(k_{\perp}\right)=\mathcal{G}\left(k_{x}, k_{y}\right)$, and $\Delta k=k_{\|}(\Omega)-\Omega / v_{g}$ is the wavevector mismatch between the generated $\mathrm{THz}$ wave and the incident optical wave.

In Eq. (8), three terms play a key role concerning the shape of the emitted $\mathrm{THz}$ spectrum and the way it is related to the NIR pulse shape: the phase mismatch factor $\operatorname{sinc}\left[L_{e} \Delta k / 2\right], C(\Omega)$ and $\mathcal{G}\left(k_{\perp}\right)$. Here, we will discuss only the influence of the sinc term, the discussion concerning $C(\Omega)$ and $\mathcal{G}\left(k_{\perp}\right)$ being postponed to sections 3 and 4 respectively. The phase mismatch factor gives its main contribution to the THz spectrum for the frequency $\Omega$ such that $\Delta k(\Omega)=0$, the so-called phase matching condition. Assuming that the NIR pulse is almost a plane wave across the crystal, i.e. $k_{\perp} \simeq 0$, this latter condition may be written:

$$
\Delta k=k(\Omega)-\frac{\Omega}{v_{g}}=\Omega\left(\frac{1}{v_{\varphi}}-\frac{1}{v_{g}}\right)=0,
$$

where $v_{\varphi}=c / n(\Omega)$ is the phase velocity of the THz wave. Thus, the phase matching condition sets that:

$$
v_{\varphi}(\Omega)=v_{g}\left(\omega_{0}\right) \Longleftrightarrow n(\Omega)=n_{g}\left(\omega_{0}\right) .
$$

Dispersion of ZnTe in the NIR range is well describe by the following Sellmeier equation [25]:

$$
n^{2}(\lambda)=4.27+\frac{3.01 \lambda^{2}}{\lambda^{2}-0.142}
$$

where $\lambda$ is in $\mu \mathrm{m}$, and in the THz range by the dielectric function given in [12]:

$$
\tilde{\varepsilon}(\Omega)=(n+i \kappa)^{2}=\varepsilon_{\mathrm{el}}+\frac{\varepsilon_{\mathrm{st}} \Omega_{\mathrm{TO}}^{2}}{\Omega_{\mathrm{TO}}^{2}-\Omega^{2}-i 2 \gamma \Omega},
$$

with $\varepsilon_{\mathrm{el}}=7.44, \varepsilon_{\mathrm{st}}=2.58, \Omega_{\mathrm{TO}} / 2 \pi=5.32 \mathrm{THz}$ and $\gamma / 2 \pi=0.022 \mathrm{THz}$. So, a NIR pulse with a spectrum centered at $\lambda_{0} \sim 800 \mathrm{~nm}$ leads to a THz wave centered around $2.5 \mathrm{THz}$ (see, for example, the black curve of figure 2b). Actually, the way the phase mismatch factor affects the shape of the THz spectrum depends on two factors: $\lambda_{0}$ and $L_{e}$ [1]. As a consequence, it offers little possibilities to generate shaped $\mathrm{THz}$ spectra with an easy tunability. However, we will see that $C(\Omega)$ and $\mathcal{G}\left(k_{\perp}\right)$ are more promising candidates for this purpose.

Finally, in order to compare the experimental results presented below with the theoretical predictions of Eq. (8), we have to take into account the detection of the THz electric field via 
electro-optic (EO) sampling in a second $\langle 110\rangle \mathrm{ZnTe}$ crystal, with a thickness $L_{d}$, using a weak probe NIR ultrashort pulse. To this end, we use the expression of the EO signal given in [11]:

$$
S(\tau) \propto \int_{-\infty}^{+\infty} E_{\mathrm{THz}}\left(\Omega, k_{\perp}, L_{e}\right) f(\Omega) e^{-i \Omega \tau} d \Omega=\int_{-\infty}^{+\infty} S(\Omega) e^{-i \Omega \tau} d \Omega,
$$

where $\tau$ is the time delay between the THz and the NIR pulses, $S(\Omega)$ is the spectral amplitude of the EO signal, and

$$
f(\Omega)=C_{\text {probe }}(\Omega) \chi^{(2)}(\Omega) \frac{e^{i L_{d} \Delta k}-1}{i \Delta k},
$$

$C_{\text {probe }}(\Omega)$ being the power spectrum of the probe pulse.

Furthermore, in Eqs. (8) and (14), we will assume that the frequency dependence of $\chi^{(2)}$ is given by [39]:

$$
\chi^{(2)}(\Omega)=2 d_{14}\left[1+C\left(1-\frac{\Omega}{\Omega_{\mathrm{TO}}}\right)^{-1}\right],
$$

where $d_{14}=90 \mathrm{pm} / \mathrm{V}$ and $C=-0.07$.

\section{Generation of tunable THz pulses by optical pulse shaping in the spectral domain}

\subsection{Further analysis of THz generation by optical rectification I}

In section 2, we have shown the influence of the phase mismatch factor on the THz spectrum. Here, we are going to study in which way one can control the shape of the THz pulse by acting on the power spectrum $C(\Omega)$. To this end, we assume that the NIR pulse is a plane wave in the crystal, so that $k_{\perp}=0$ and $\mathcal{G}\left(k_{\perp}\right)=1$ in Eq. (8). With these assumptions, it is clear that, apart from the sinc term, this is the power spectrum, i.e. the temporal pulse shape of the incident NIR pulse, since $C(\Omega)=\int|\mathcal{E}(t)|^{2} \exp (i \Omega t) d t$, that controls the shape of $E_{\mathrm{THz}}\left(\Omega, L_{e}\right)$. Actually, Eq. (5) clearly shows that $C(\Omega)$ strongly depends on the NIR pulse spectrum and more especially on the relative phase $\phi(\omega)-\phi(\omega-\Omega)$ between all frequency pairs within the pulse separated by $\Omega$. So, by acting on the spectral phase $\phi(\omega)$ of the NIR pulse in the appropriate way it is possible to finely control $C(\Omega)$ and, consequently, the THz spectrum shape.

In what follows, we will show some examples of tunable narrow-band $\mathrm{THz}$ spectra, the tunability being achieved through a sinusoidal spectral phase modulation and the spectral narrowing through an additional triangular phase.

\subsection{Experimental setup}

The experimental setup is depicted on Fig. 1: a Ti:sapphire chirped pulse amplifier (Femtopower Compact Pro) delivering laser pulses of duration $\tau_{\mathrm{p}}=35 \mathrm{fs}$ at $790 \mathrm{~nm}$ with a $1 \mathrm{kHz}$ repetition rate is used to generate and detect $\mathrm{THz}$ waves. About $90 \%$ of the output of the CPA is tailored by a pulse shaper and is used for the generation of $\mathrm{THz}$ pulses by optical rectification in a $\langle 110\rangle$ ZnTe crystal with a thickness $L_{e}\left(L_{e}=300 \mu \mathrm{m}\right.$ for the experiments of section 3.3 and $1 \mathrm{~mm}$ for those of sections 3.4 and 3.5).

The pulse shaper consists in a half-zero-dispersion line made of a $600 \mathrm{~g} / \mathrm{mm}$ grating and a $f=600 \mathrm{~mm}$ cylindrical mirror that spatially disperses and focuses all the spectral components 


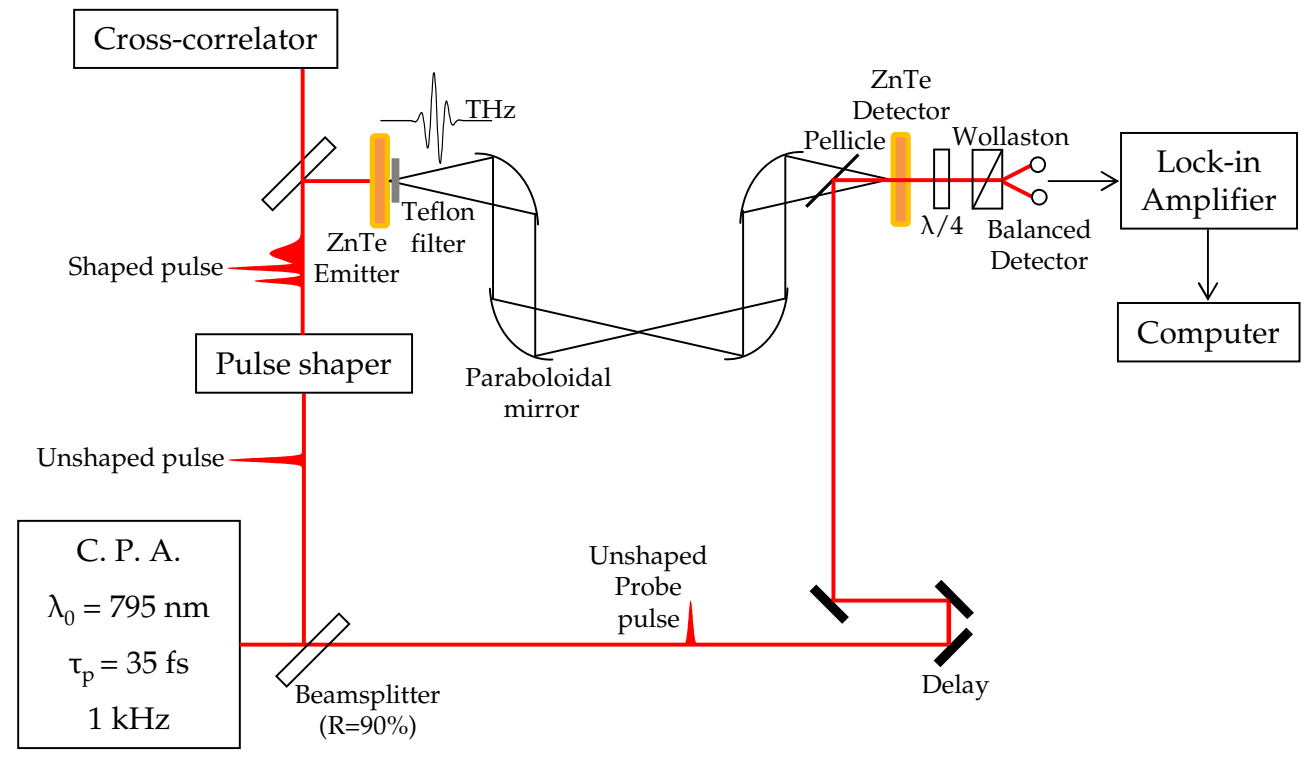

Figure 1. Experimental setup for the generation of tunable THz pulses by optical pulse shaping in the spectral domain.

of the pulse in the Fourier plane where a programmable single liquid crystal spatial light modulator (LC SLM) with 640 pixels, manufactured by Jenoptik AG, acts as a spectral filter $[22,23,33,42]$. A plane mirror positioned just after the SLM folds the line to perform an inverse Fourier transform and reassemble all the spectral components of the shaped pulse. The SLM is such that it is only possible to change the spectral phase of the incoming pulse. The intensity profile of the shaped laser pulse is measured by cross-correlation, in a type I $\mathrm{BBO}$ crystal, of this pulse with an unshaped reference pulse.

The beam exiting from the pulse shaper then excites the ZnTe emitter at normal incidence. After removal of the residual NIR pulse by a Teflon filter, the generated $\mathrm{THz}$ wave is imaged into a second $\langle 110\rangle \mathrm{ZnTe}$ crystal with a thickness $L_{d}$ by means of four off-axis paraboloidal mirrors $\left(L_{d}=300 \mu \mathrm{m}\right.$ for the experiments of section 3.3 and $500 \mu \mathrm{m}$ for those of sections 3.4 and 3.5). There, it is measured via electro-optic effect using a weak unshaped NIR pulse coming directly from the exit of the Ti:sapphire amplifier [11]. By varying the delay $\tau$ between the $\mathrm{THz}$ pulse and the ultrashort NIR pulse, one gets an EO signal, $S(\tau)$, corresponding to a cross-correlation between the laser pulse and the THz wave integrated over the EO crystal length. This signal is then recorded by ellipsometry (the ellipsometer consists in a $\lambda / 4$ wave plate, a Wollaston polarizer, and two balanced detectors).

\subsection{Sinusoidal phase modulation: generation of $\mathrm{THz}$ pulse trains}

It is well known that selectivity in the $\mathrm{THz}$ spectral range can be achieved by means of terahertz-rate sequences of femtosecond pulses [1,21,41]. Experimentally, such a pulse train with a repetition rate $F_{\text {rep }}$ is generated by applying a periodic phase modulation with a period $F_{\text {rep }}$, the most used being $\phi(\omega)=a \sin \left(\omega / F_{\text {rep }}\right)$. In the absence of any phase modulation, i.e. 

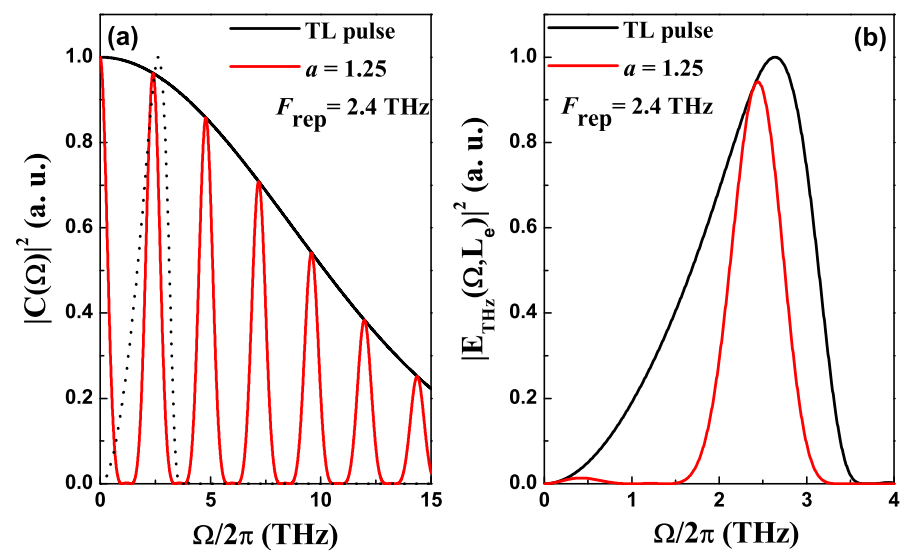

Figure 2. (a) Power spectrum of an optical pulse with $\lambda_{0}=790 \mathrm{~nm}$, and a pulse duration $\tau_{p}=35 \mathrm{fs}$ : transform-limited (TL) pulse (black curve), shaped pulse with a sinusoidal phase modulation $\phi(\omega)=a \sin \left(\omega / F_{\text {rep }}\right)$ (red curve). The dotted curve superimposed to $|C(\Omega)|^{2}$ corresponds to the $\mathrm{THz}$ spectrum generated by a TL pulse. (b) Terahertz spectra generated by optical rectification in a $\langle 110\rangle \mathrm{ZnTe}$ of a: TL pulse (black curve), sinusoidally phase modulated pulse (red curve).

for a transform-limited pulse corresponding to $\phi(\omega)=0$, the power spectrum is a bell-shaped curve centered on $\Omega=0$, with a half-width at maximum equal to the pulse bandwidth (black curve, Fig. 2a). However, if a sinusoidal phase modulation is applied, then $C(\Omega)$ exhibits spectral interference fringes with maxima centered at the frequencies $\Omega_{n}=2 \pi n F_{\text {rep }}$ (where $n$ is an integer) (red curve, Fig. 2a, where $a=1.25$ and $F_{\text {rep }}=2.4 \mathrm{THz}$ ). Now, if one takes into account the spectral filtering of the powerspectrum induced by the phase matching condition, corresponding to a multiplication of $|C(\Omega)|^{2}$ by the dotted curve of Fig. $2 \mathrm{a}$, it results in a narrower THz spectrum centered at the frequencies $\Omega_{n}$ within the THz band authorized by the phase matching condition (Fig. 2b). This spectral narrowing can also be understood by a reasoning in the time domain. Actually, each pulse of the NIR pulse train, two consecutive pulses being separated by $T_{\text {rep }}=1 / F_{\text {rep }}$, leads to its own $\mathrm{THz}$ waveform. All these waveforms add coherently to generate a $\mathrm{THz}$ train with a repetition rate $F_{\text {rep }}$ whose spectrum precisely consists in peaks centered at frequencies $\Omega_{n}=2 \pi n / T_{\text {rep }}$, the bandwidth of these peaks decreasing as the number of pulses in the $\mathrm{THz}$ train increases.

Fig. 3a displays the EO signals, $S(\tau)$, associated to THz trains generated with our setup for different values of $F_{\text {rep }}$ and $a=1.25$, whereas the corresponding spectra, defined as $|S(\Omega)|^{2}$, are represented in Fig. 3c. In agreement with the former analysis, we observe a THz pulse train with a spectrum that can be tuned within the spectral range obtained for an unshaped pulse by adjusting the repetition rate of the NIR pulse train. There is a very good agreement between our results and the spectra calculated with the model described in section 2 (Fig. 3c and $\mathrm{d}$ ). These two last figures clearly show that we can tune the carrier frequency of $\mathrm{THz}$ pulse simply by changing the repetition rate of the NIR pulse train. 


\section{Experiment}

(a)

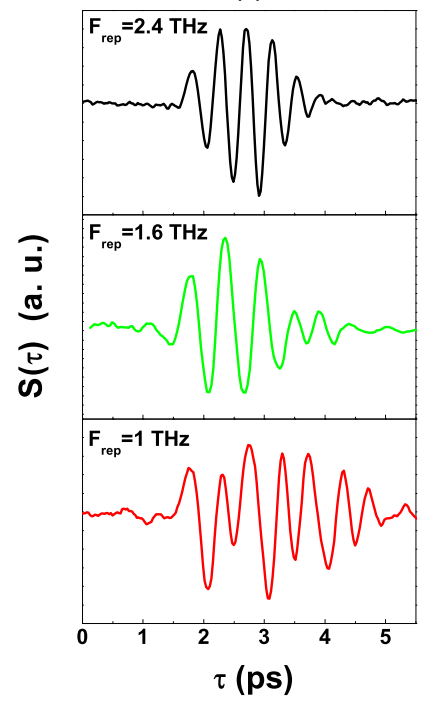

(c)

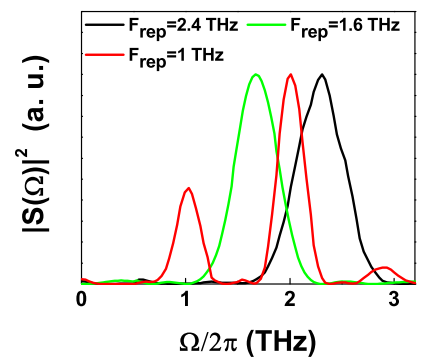

Simulation

(b)

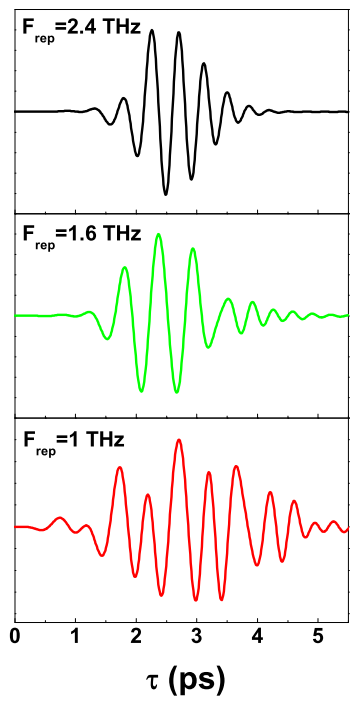

(d)

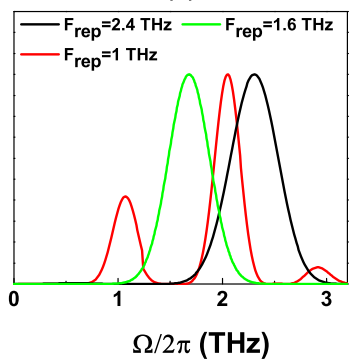

Figure 3. THz pulse train with an adjustable repetition rate $F_{\text {rep }}$, the paramater $a$ being equal to 1.25 . Experiment: (a) waveforms and (c) corresponding spectra. Simulation: (b) waveforms and (d) corresponding spectra (taken from [37]).

However, for $F_{\text {rep }}=1 \mathrm{THz}$, one can note that the spectrum also displays second and third-harmonic peaks centered at 2 and $3 \mathrm{THz}$ respectively. They are inherent to the use of a sinusoidal phase modulation: for $F_{\text {rep }}=1 \mathrm{THz}$, the phase matching condition is less selective with respect to the peaks of the power spectrum, leading to these harmonics. From a "temporal" point of view, these harmonics arise because, for this repetition rate, the $\mathrm{THz}$ waveform generated by each NIR pulse of the train has a duration shorter or comparable to $T_{\text {rep. }}$. Thus, when all these partial waveforms add coherently to generate the total $\mathrm{THz}$ electric field $E_{\mathrm{THz}}(t)$, they leads to an overall shape that is more complex and damps more slowly than the sinusoidal waveform obtained for $F_{\text {rep }}=1.6$ or $2.4 \mathrm{THz}$. Thanks to the higher 

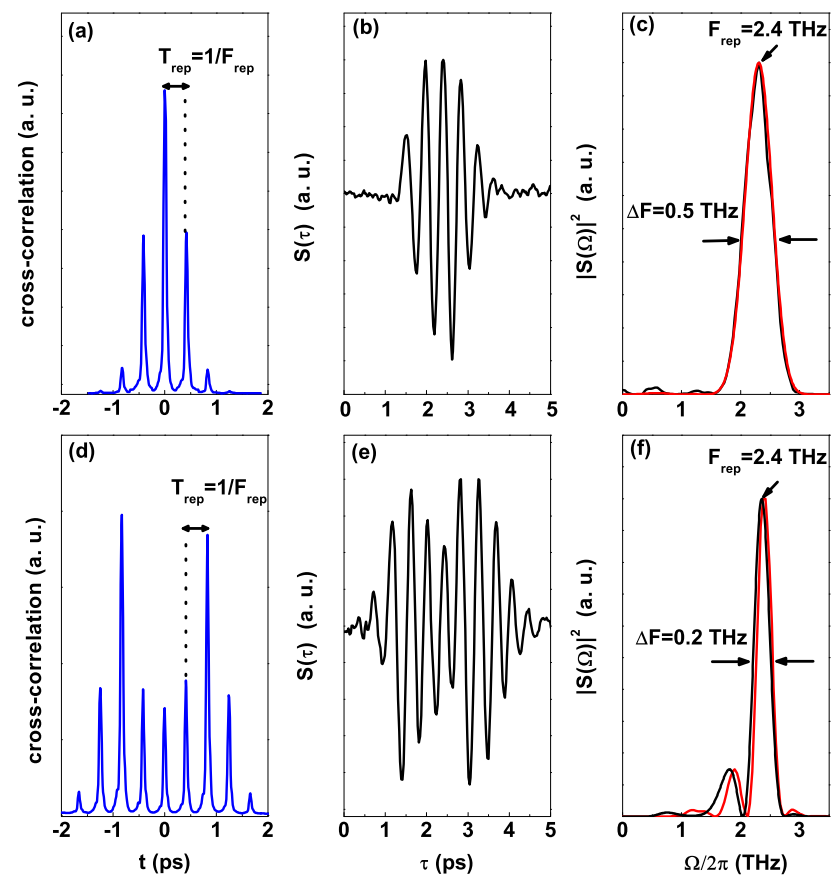

Figure 4. Experimental illustration of the spectral narrowing by a sinusoidal phase modulation $\phi(\omega)=a \sin \left(\omega / F_{\text {rep }}\right)$ : (a) cross-correlation of the optical pulse train generated with $a=1.25$ and $F_{\text {rep }}=2.4 \mathrm{THz}$. (b) Associated THz train and (c) corresponding spectrum: experiment in black and simulation in red. (d) cross-correlation of the NIR pulse train generated with $a=2.65$ and $F_{\text {rep }}=2.4 \mathrm{THz}$. (e) Associated THz train and (f) corresponding spectrum (taken from [37]).

number of oscillations, that is to the longer $\mathrm{THz}$ pulse duration, the width of the harmonics for $F_{\text {rep }}=1 \mathrm{THz}$ is also reduced $(\sim 280 \mathrm{GHz})$ compared to the spectra for $F_{\text {rep }}=1.6$ or $2.4 \mathrm{THz}$ (bandwidth $\sim 500 \mathrm{GHz}$ ).

To see if it is possible to further decrease the bandwidth of the $\mathrm{THz}$ spectrum when a sinusoidal phase modulation is applied to the rectified NIR pulse, let us consider a transform-limited laser pulse with a duration $\tau_{\mathrm{p}}$, and a gaussian amplitude

$$
A(\omega)=E_{0} T_{p} \sqrt{\pi} \exp \left(-\frac{\omega^{2} T_{p}^{2}}{4}\right), T_{p}=\tau_{p} / \sqrt{2 \ln 2} .
$$

When a sinusoidal phase $\phi(\omega)$ is applied to this pulse, it results in a pulse train with a temporal dependence of the electric field given by [29]:

$$
\mathcal{E}(t) \propto \sum_{m=-\infty}^{+\infty} J_{m}(a) \exp \left(\frac{i m \pi}{2}\right) \exp \left[-\left(\frac{t-m T_{\text {rep }}}{T_{\mathrm{p}}}\right)^{2}\right],
$$


where the $J_{m}$ are Bessel functions of the first kind. This equation shows that, whatever the phase modulation period $F_{\text {rep }}$, the amplitude of the $m^{\text {th }}$ pulse of the train is given by $\left|J_{m}(a)\right|$. So, by playing on the modulation strength $a$ one can control, to some extend, the weight of the different pulses of the train. More precisely, the evolution of $\left|J_{m}(a)\right|$ is the following [29]: except for $\left|J_{0}\right|$, it increases when $a$ is varied from 1.25 to 2.65 , leading to an enhancement of the number of pulses in the train. Hence, increasing $a$ will lengthen the $\mathrm{THz}$ pulse duration and lead to a narrower spectrum, this regardless of the repetition rate $F_{\text {rep. }}$ Fig. 4 f displays the $\mathrm{THz}$ spectrum obtained by applying a sinusoidal phase modulation with $a=2.65$ and $F_{\text {rep }}=$ $2.4 \mathrm{THz}$. With this modulation strength, there are nine pulses in the NIR pulse sequence (Fig. $4 \mathrm{~d}$ ) instead of five for $a=1.25$ (Fig. 4a), giving a spectrum with a bandwidth of $0.2 \mathrm{THz}$. Here again, experiment and simulation are in good agreement (Fig. 4f).

Nevertheless, one also observes some ripples on the spectrum of Fig. 4f. They can be attributed to the difference in amplitude between the different pulses of the NIR pulse train, and especially to the two main peaks centered around $\pm 800 \mathrm{fs}$ in Fig. 4d, resulting from the fact that, for $a=2.65,\left|J_{ \pm 2}(a)\right|>\left|J_{ \pm n}(a)\right|(|n| \neq 2)$. Unfortunately, their amplitude cannot be modified easily without affecting the amplitude of the other peaks. Indeed, as shown in reference [29], it is impossible to produce pulse trains with more than three pulses of equal amplitude with a simple sinusoidal phase. On the other hand, one can solve this problem by adding higher harmonic orders to the applied phase modulation, i.e. by means of the following spectral phase: $\phi(\omega)=\sum_{n} F_{n} \sin \left(n \omega / F_{\text {rep }}+\phi_{n}\right)$. However, this phase is harder to implement since it requires the use of an optimization algorithm to find the best value for the weight $F_{n}$ of each harmonic. In the same spirit, Ahn et al. [1] have used a Gerchberg-Saxton algorithm to find the best spectral phase in order to generate many nearly equally spaced identical optical pulses. But, here again, this method relies on the ability of the algorithm to converge towards the expected solution. Finally, we will show in section 3.5 that it is possible to shrink the $\mathrm{THz}$ pulse bandwidth simply by adding a soundly chosen triangular phase modulation to the sinusoidal one. Before that, we will consider the effect of a triangular phase modulation on the $\mathrm{THz}$ generation.

\subsection{Triangular phase modulation: generation of phase-locked THz pulse pairs}

Let us consider a triangular phase modulation defined by

$$
\phi(\omega)=-\Delta \tau|\omega-\delta \omega|,
$$

where $\Delta \tau$ is the slope of the spectral phase, and $\delta \omega$ is the detuning with respect to the center of the spectral amplitude $\mathcal{E}(\omega)$. Such a phase, when it is applied to a transform-limited pulse with a duration $\tau_{p}$ and a gaussian shape

$$
\mathcal{E}(t)=E_{0} \exp \left[-\left(\frac{t}{T_{p}}\right)^{2}\right], T_{p}=\tau_{p} / \sqrt{2 \ln 2},
$$

leads to a pair of pulses whose temporal amplitude is given by [29]:

$$
\mathcal{E}(t)=\mathcal{E}_{+}(t+\Delta \tau)+\mathcal{E}_{-}(t-\Delta \tau),
$$

with

$$
\mathcal{E}_{ \pm}(t)=\frac{E_{0}}{2} e^{-\left(t / T_{p}\right)^{2}}\left[1-\operatorname{erf}\left(i \frac{t}{T_{p}} \pm \frac{T_{p} \delta \omega}{2}\right)\right]
$$


with the error function, erf, defined by

$$
\operatorname{erf}(x)=\frac{2}{\sqrt{\pi}} \int_{0}^{x} \exp \left(-y^{2}\right) d y .
$$

So it leads to two pulses separated by a time delay $t_{p}=2 \Delta \tau$, with a ratio of their amplitudes $\left(\mathcal{E}_{+}(0) / \mathcal{E}_{-}(0)\right)$ controlled by the detuning $\delta \omega$, this ratio being equal to 1 for $\delta \omega=0$. This kind of sequence of ultrashort phase-locked pulses has been used extensively to perform temporal coherent control experiments with optical pulses [3]. It has also been used successfully in the terahertz range to control various processes [15, 16, 44] (see also section 3.5 for a brief presentation). In these latter experiments, the THz pulse pair was generated from a NIR pulse pair created by a Michelson or a Mach-Zender interferometer. Here, we will demonstrate that it is possible to generate a $\mathrm{THz}$ pulse pair by applying a triangular spectral phase to the rectified NIR pulse. Since temporal coherent control experiments require a balanced pair of pulses to achieve the best efficiency, we will only consider, in what follows, the case where $\delta \omega=0$, so that $\mathcal{E}_{+}(t)=\mathcal{E}_{-}(t)$.
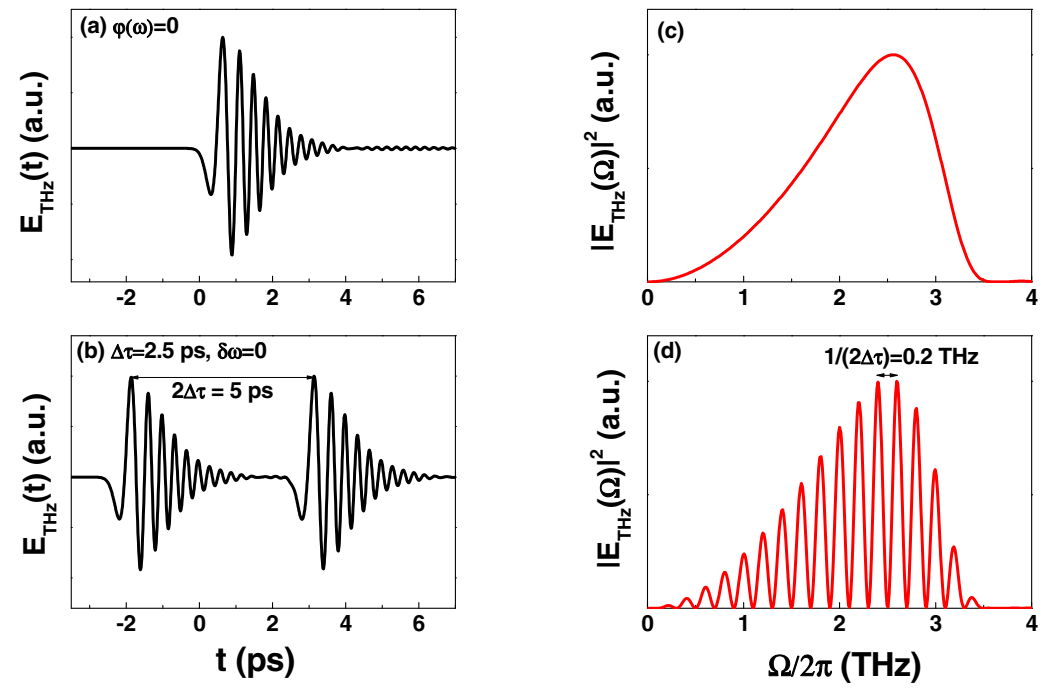

Figure 5. THz waveforms generated by optical rectification in a $300 \mu$ m-thick ZnTe crystal of: (a) a transform-limited NIR pulse, (b) a NIR pulse having a triangular phase modulation with $\Delta \tau=2.5 \mathrm{ps}$ and $\delta \omega=0$. (c) and (d) respectively show the spectra of (a) and (b). In (d), the fringe spacing is $1 / t_{p}=0.2 \mathrm{THz}$.

By optical rectification of a pair of balanced NIR pulses in a ZnTe crystal, one obtains a $\mathrm{THz}$ pulse pair with the following electric field:

$$
E_{\mathrm{THz}}(t)=E_{\mathrm{THz}_{+}}(t+\Delta \tau)+E_{\mathrm{THz}_{+}}(t-\Delta \tau),
$$

where $E_{\mathrm{THz}_{+}}(t)$ is the electric field of the THz pulse generated by optical rectification of a NIR pulse whose temporal amplitude is $\mathcal{E}_{+}(t)$. Its amplitude spectrum is given by

$$
\begin{aligned}
E_{\mathrm{THz}}(\Omega) & =E_{\mathrm{THz}_{+}}(\Omega) e^{-i \Omega \Delta \tau}+E_{\mathrm{THz}_{+}}(\Omega) e^{i \Omega \Delta \tau} \\
& =E_{\mathrm{THz}_{+}}(\Omega) e^{-i \Omega \Delta \tau}\left(1+e^{i \Omega t_{p}}\right),
\end{aligned}
$$


so that the expected spectrum of the EO signal is

$$
|S(\Omega)|^{2} \propto\left|E_{\mathrm{THz}}(\Omega)\right|^{2}=2\left|E_{\mathrm{THz}_{+}}(\Omega)\right|^{2}\left[1+\cos \left(\Omega t_{p}\right)\right] .
$$

It has the same enveloppe as the spectrum $\left|E_{\mathrm{THz}_{+}}(\Omega)\right|^{2}$ of a single $\mathrm{THz}$ pulse (shown on Fig. 5c), but with modulations due to the spectral interference of the two $\mathrm{THz}$ pulses, the fringes spacing being $1 / t_{p}$ (see Fig. $5 d$ ).

Fig. 6 shows the $\mathrm{THz}$ pulse pair generated experimentally by applying a triangular phase modulation with $\Delta \tau=2.5 \mathrm{ps}$ and $\delta \omega=0$ to the NIR pulse (the same parameters as in the simulation of Fig. 5). As mentioned previously, for $\delta \omega=0$, both NIR pulses are balanced (Fig. $6 \mathrm{~d}$ ), and give rise to a pair of $\mathrm{THz}$ pulses with the same carrier frequency and separated by $t_{p}=5$ ps (Fig. 6e). The corresponding EO signal spectrum (Fig. 6f) has the same enveloppe as the EO signal spectrum of a single $\mathrm{THz}$ pulse (Fig. 6c), but exhibits oscillations with a period $1 / t_{p}=0.2 \mathrm{THz}$.

However, in addition to the NIR pulse pair, the cross-correlation trace of Fig. $6 \mathrm{~d}$ also has an unwanted TL pulse centered at $t=0$. This distortion is due to the combination of two limitations of the LC SLMs [35]: first, since LC SLMs have a phase operating range slightly above $2 \pi$, the applied phase modulation is "wrapped" to be within the $[0,2 \pi]$ interval
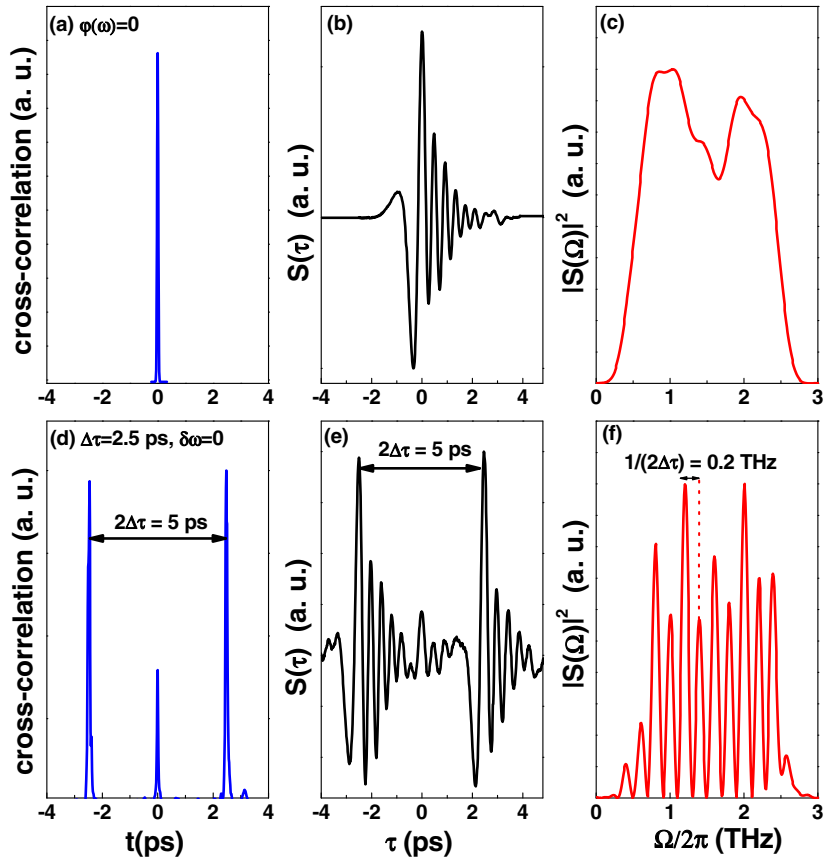

Figure 6. Sequence of $\mathrm{THz}$ phase-locked pulses experimentally generated by applying a triangular phase modulation with $\Delta \tau=2.5 \mathrm{ps}$ and $\delta \omega=0$ to the rectified NIR pulse: (a) cross-correlation of the initial TL NIR pulse, (b) associated THz waveform and (c) corresponding spectrum. (d) cross-correlation of the NIR pulse pair, (e) associated THz waveform and (f) corresponding spectrum (taken from [37]). 
[33]. Second, the pixels, and consequently the gaps, of the LC SLM are not sharply defined, leading to some smoothing of the pixelated phase. Combined together these limitations lead to the apparition of "modulator replica" pulses in addition to the desired pulse shape. In our experiment, the phase varies from $-223 \pi$ to 0 over the first half of the spectrum and, symetrically, from 0 to $-223 \pi$ over the second half. This leads to numerous phase-wraps and, in combination with the smoothed spatial response of the pixels, gives the pulse centered at $t=0$ in Fig. $6 \mathrm{~d}$. This unwanted effect can be avoided by the use of a diffraction-based pulse shaper [36]. Nevertheless, as we will see in the next section, it does not affect significantly the $\mathrm{THz}$ spectrum shape.

\subsection{Sinusoidal + triangular phase: tunable narrow band THz pulses.}

We now consider the action of both phases introduced previously, i.e.

$$
\phi(\omega)=a \sin \left(\omega / F_{\text {rep }}\right)-\Delta \tau|\omega-\delta \omega| .
$$

According to sections 3.3 and 3.4, we expect a sequence of pulse trains with a repetition rate

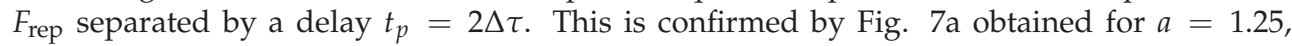
$F_{\text {rep }}=2 \mathrm{THz}, \Delta \tau=2.5 \mathrm{ps}$ and $\delta \omega=0$. As in section 3.4, the applied triangular phase modulation leads to numerous phase-wraps and, in combination with the limitations of the LC SLM, gives an additional TL pulse centered at $t=0$. This undesired pulse and the pulse train sequence generate their own $\mathrm{THz}$ waveforms that add coherently (Fig. $7 \mathrm{~b}$ ). The $\mathrm{THz}$ spectrum is displayed on Fig. 7c. Due to the triangular phase modulation, it still exhibits interference fringes with a spacing equal to $1 / t_{p}$, but, thanks to the sinusoidal phase, it is

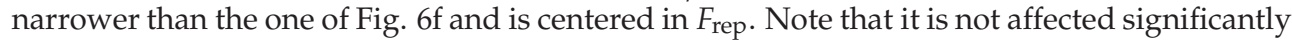
by the THz pulse generated by the TL pulse due to the limitations of the SLM as can be seen from the simulation of Fig. $7 \mathrm{f}$ which does not include these latter.

These $\mathrm{THz}$ pulse trains sequences are of great importance for temporal coherent control and the generation of tunable narrow-band $\mathrm{THz}$ pulses. The goal of temporal coherent control is to excite selectively some levels of a quantum system by means of a pair of ultrashort phase-locked pulses in order to steer the evolution of this system towards a desirable state through its interaction with light (for example to control the outcome of a photo-induced chemical reaction). Let us consider, for example, a quantum system with a ground state $|g\rangle$ and $N$ excited states $\left|e_{k}\right\rangle$ (with energies $\hbar \Omega_{k}$ ), which is excited by a sequence of two identical ultrashort pulses separated by a delay $t_{p}$. We suppose that the bandwidth of the pulses is broad enough to excite the $N$ states $\left|e_{k}\right\rangle$. In the weak field regime, the state vector $|\Psi(t)\rangle$ of the system after interaction with this sequence of pulses is given by [3]:

$$
|\Psi(t)\rangle=|g\rangle+\sum_{k=1}^{N} b_{k} e^{-i \Omega_{k} t}\left|e_{k}\right\rangle,
$$

with

$$
b_{k}=\frac{1}{i \hbar} \mu_{k g} E_{t o t}\left(\Omega_{k}\right),
$$

where $\mu_{k g}$ is the transition dipole moment from state $|g\rangle$ to state $\left|e_{k}\right\rangle$, and $E_{\text {tot }}\left(\Omega_{k}\right)$ is the spectral amplitude of the total electric field taken at frequency $\Omega_{k}$, given by

$$
E_{t o t}\left(\Omega_{k}\right)=E\left(\Omega_{k}\right)\left(1+e^{i \Omega_{k} t_{p}}\right)
$$



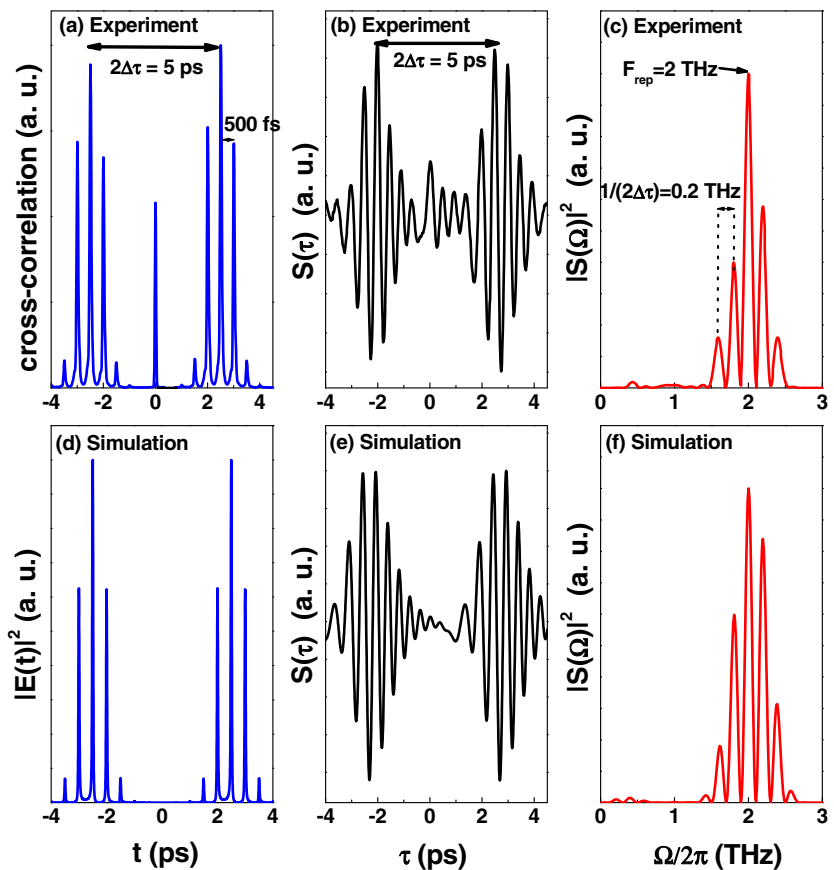

Figure 7. Sequence of $\mathrm{THz}$ pulse trains generated by applying simultaneously a triangular and a sinusoidal phase with $a=1.25, F_{\text {rep }}=2 \mathrm{THz}, \Delta \tau=2.5$ ps and $\delta \omega=0$. (a) cross-correlation of the NIR pulse, (b) associated THz waveform and (c) corresponding spectrum. Panels (d), (e) and (f) correspond respectively to the simulations of panels (a), (b) and (c) (taken from [37]).
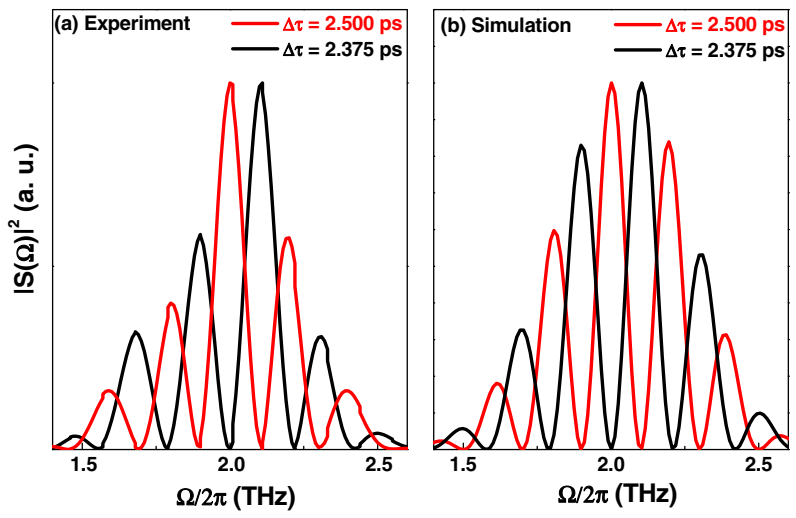

Figure 8. "In-phase" and "out-of-phase" sequences of pulse trains at $\Omega_{k} / 2 \pi=2 \mathrm{THz}$ : (a) Experimental $\mathrm{THz}$ spectra obtained for $a=1.25, F_{\text {rep }}=2 \mathrm{THz}, \delta \omega=0, \Delta \tau=2.5 \mathrm{ps}$ (red curve, corresponding to $\Delta \varphi=2 \pi p$ ) and $\Delta \tau=2.375$ ps (black curve, corresponding to $\Delta \varphi=2 \pi(p+1 / 2)$ ). (b) Corresponding simulations (taken from [37]). 

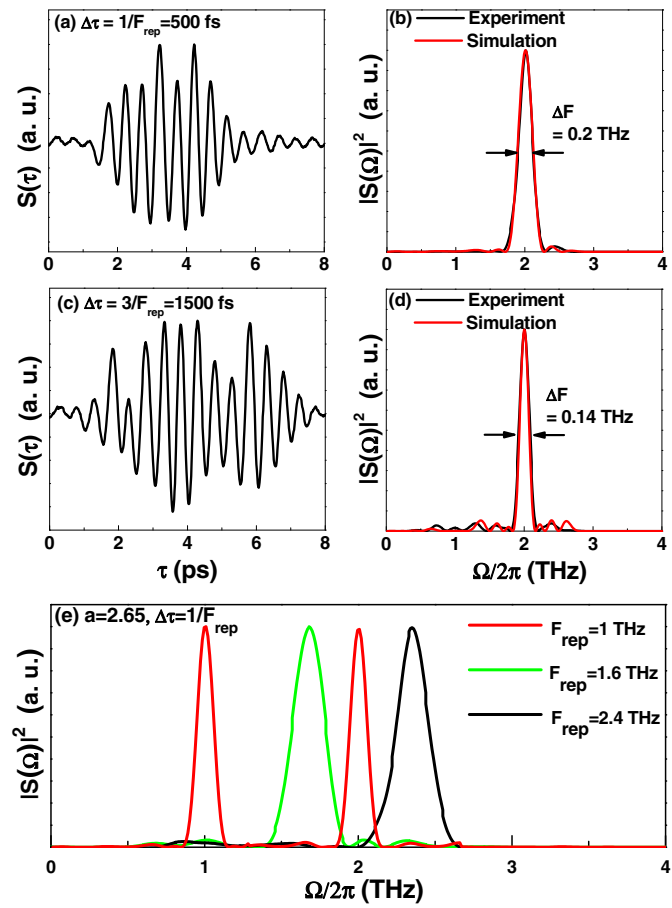

Figure 9. Spectral smoothing ((a) and (b)) and spectral narrowing ((c) and (d)) with a sequence of $\mathrm{THz}$ pulse trains. In both cases $a=2.65, F_{\text {rep }}=2 \mathrm{THz}$ and $\delta \omega=0$. (e) Experimental illustration of the tunability of the spectral smoothing shown in (a): for all the curves $a=2.65, \Delta \tau=1 / F_{\text {rep }}$ and $\delta \omega=0$ (taken from [37]).

$E(\Omega)$ being the amplitude spectrum of a single pulse. Thus, the probability to excite the state $\left|e_{k}\right\rangle$ is

$$
\left|\left\langle e_{k} \mid \Psi(t)\right\rangle\right|^{2}=\left|b_{k}\right|^{2} \propto\left|E\left(\Omega_{k}\right)\right|^{2}\left[1+\cos \left(\Omega_{k} t_{p}\right)\right] .
$$

This probability strongly depends on the relative phase $\Delta \varphi\left(\Omega_{k}\right)=\Omega_{k} t_{p}$ between the two pulses of the sequence: the excitation is "constructive" for $\Delta \varphi=2 \pi p$ ( $p$ is an integer) and "destructive" for $\Delta \varphi=2 \pi(p+1 / 2)$. The fine tuning of $\Delta \varphi$ is achieved via the tuning of the delay $t_{p}$, and strongly depends on its (interferometric) stability. In the THz range, our setup makes it possible to generate easily these "in-phase" and "out-of-phase" pulse pairs, simply by changing the slope $\Delta \tau$ of the triangular phase. For instance, Fig. 8 shows two $\mathrm{THz}$ pulse sequences leading respectively to a constructive excitation (red curve) and a destructive excitation (black curve) at $\Omega_{k} / 2 \pi=2 \mathrm{THz}$.

One can also take advantage of the combined triangular and sinusoidal phases to generate less structured $\mathrm{THz}$ spectra than the one of Fig. 4f, simply by choosing a delay between the $\mathrm{THz}$ pulses shorter than their duration. Then the THz waveforms are superposed and, for a suitably choosen value of $\Delta \tau$, give rise to a single $\mathrm{THz}$ train with pulses having almost the same amplitude, resulting in a spectrum with a well defined peak, centered at the pulse train repetition rate $F_{\text {rep. }}$ This remarkable value of $\Delta \tau$ is $1 / F_{\text {rep }}, \forall F_{\text {rep. }}$. Figures $9 \mathrm{a}$ and $9 \mathrm{~b}$ display 
an example of such a "smooth" narrow-band THz spectrum for $F_{\text {rep }}=2 \mathrm{THz}$, whereas Fig. $9 \mathrm{e}$ shows the tunability of this method. On this figure, one can see that, contrary to Fig. 3c, the peaks at 1 and $2 \mathrm{THz}$ for $F_{\text {rep }}=1 \mathrm{THz}$ have the same amplitude. It results from the fact that this spectrum was obtained with a thicker crystal than the one used in section 6, leading to an unshaped THz spectrum with almost the same energy around 1 and $2 \mathrm{THz}$ (see Fig. 6c) unlike the unshaped spectrum corresponding to Fig. 3. Now, for a given value of $F_{\text {rep }}$, if one tunes $\Delta \tau$ around $1 / F_{\text {rep }}$, it is then possible to generate relatively clean $\mathrm{THz}$ spectra with a narrower bandwidth. An example is given in figures $9 \mathrm{c}$ and $9 \mathrm{~d}$ : for $\Delta \tau=3 / F_{\text {rep }}=1.5 \mathrm{ps}$, the $\mathrm{THz}$ waveform exhibits more oscillations than in Fig. 9a, leading to a spectrum with a bandwidth of $140 \mathrm{GHz}$. However, for repetition rates like $F_{\text {rep }}=1 \mathrm{THz}$, one should note that although this method makes it possible to narrow and smooth the peak at $1 \mathrm{THz}$ and its harmonics, it cannot suppress these latter since they are inherent to the use of a sinusoidal phase modulation.

\subsection{Limitations of this method}

In this section, we will address some points concerning the limitations of our approach and the possibility to generate even narrower $\mathrm{THz}$ spectra.

First, we will consider the limitations imposed by the pulse shaper. We have already met such limitations in sections 3.4 and 3.5, leading to the apparition of "modulator replica" pulses in addition to the desired pulse shape. Now, we will see that the pixelated nature of the LC SLM imposes upper limits to $T_{\text {rep }}$ and $\Delta \tau$. Indeed, to be correctly sampled by the modulator's pixels, and thus avoid temporal aliasing, the phase modulation must satisfy the Nyquist's criterion: the increase of phase between two adjacent pixels of the SLM must not exceed $\pi$ $[22,33,42]$. For the sinusoidal and triangular phase modulations considered separately, this criterion, applied to our setup, leads respectively to $T_{\text {rep }} \leq 4$ ps (i.e. $F_{\text {rep }} \geq 250 \mathrm{GHz}$ ) and $\Delta \tau \leq 4$ ps (i.e. $t_{p} \leq 8 \mathrm{ps}$ ). If these conditions are not satisfied, then sampling replica pulses alter the desired output waveform $[22,35]$. So, the characteristics of the pulse shaper limit the ability to generate smooth and tunable $\mathrm{THz}$ spectra at low frequencies, that is below 500 $\mathrm{GHz}$. Nevertheless, these frequencies are not of great concern here since the THz spectrum generated by optical rectification in this spectral range is almost zero.

Actually, the main limiting factor of our approach, especially if one intends to generate $\mathrm{THz}$ spectra with a bandwidth below $100 \mathrm{GHz}$, is the use of a simple sinusoidal phase modulation. As already mentioned in section 3.3, one can circumvent this limitation by adding harmonics to the applied phase, the number of harmonics increasing inversely with the required $\mathrm{THz}$ bandwidth. Indeed, an enhancement of the number of harmonics makes it possible to increase the number of pulses with the same amplitude in the NIR pulse train and, consequently, to reduce the bandwidth of the generated $\mathrm{THz}$ spectrum. However, one must not forget that the determination of the coefficients $F_{n}$ and $\phi_{n}$ requires the use of optimization methods like the one of reference [1]. Moreover, it is important to have in mind that in LC SLM pulse shapers, the tailored output pulse reflects accurately the response of the SLM over a restricted temporal window. The duration $T$ of this window is governed either by the finite spectral resolution of the zero-dispersion line or by the pixelation of the SLM (i.e. the Nyquist's criterion)[23, 35, 42]. In our setup, $T$ is limited by the pixelation of the SLM, and $T=8 \mathrm{ps}$, so the whole NIR pulse train must not exceed this duration, imposing an upper limit to the number $m$ of pulses in 
the train. Indeed, a train of $m$ pulses, with a repetition rate $F_{\text {rep }}$, has a duration $\Delta t$ given by $(m-1) / F_{\text {rep. }}$. If $\Delta t \leq T$, then

$$
m \leq m_{\max }=1+T \times F_{\text {rep }} .
$$

For $F_{\text {rep }}=0.5,1$ and $2 \mathrm{THz}, m_{\max }$ is respectively equal to 5, 9 and 17 pulses. If we consider a pulse train with a repetition rate $F_{\text {rep }}$ and a number of identical (same amplitude and same duration) pulses equaling the maximum number of pulses $m_{\max }$ authorized by the temporal window $T=8 \mathrm{ps}$ for this repetition rate, a numerical calculation of the corresponding power spectrum $C(\Omega)$ shows that the spectral interference fringes induced by this pulse train (centered at $\Omega_{n}=2 \pi n F_{\text {rep }}$ ) have a bandwidth $\sim 100 \mathrm{GHz}$, whatever the value of $F_{\text {rep. }}$. It means that, with our setup, we can't generate narrow-band $\mathrm{THz}$ pulse with a bandwidth below $100 \mathrm{GHz}$. To go below this limit, it is necessary to work with transform-limited NIR pulses having a longer duration, the number of pixels of the SLM being unchanged. Indeed, the temporal window $T$ increases as the bandwidth of the input NIR pulse decreases [35]. For example, in reference [22], the input pulse duration is $130 \mathrm{fs}$ and the LC SLM pulse shaper has a time window $T=28$ ps (the number of pixels is 640 like in our pulse shaper). With these values, it is possible to enhance $m_{\max }$, and, consequently, to generate narrow-band $\mathrm{THz}$ spectra with bandwidths down to $\sim 30 \mathrm{GHz}$ for all repetition rates as shown by numerical simulations. However, the tuning range is reduced, since working with longer NIR pulses decreases the width of the power spectrum, and consequently, the support of the THz spectrum.

\section{Generation of tunable THz pulses by optical pulse shaping in the spatial domain}

\subsection{Further analysis of $\mathrm{THz}$ generation by optical rectification II}

Our problem is to propose a way to spectrally tune the $\mathrm{THz}$ field generated by optical rectification in a zinc blende crystal by adjusting the transverse beam profile of femtosecond pump laser pulse. To solve this problem we will exploit the intermediate field properties of the emitted $\mathrm{THz}$ pulse. In order to describe the THz radiation properties in the intermediate field, we will use the Fresnel diffraction theory. To further simplify our analysis, we will consider that the beam profile has a radial symmetry and is only a function of $r$. Assuming a propagation in the $z$-direction, we will slightly modify Eq. (1) and write the electric field in the following form:

$$
E(t, x, y, z)=E(t, r, z)=u(r, z) \mathcal{E}(t) \exp \left[i\left(\omega_{0} t-k_{0} z\right)\right],
$$

where $r=\sqrt{x^{2}+y^{2}}$ and $u(r, z)$ and $\mathcal{E}(t)$ are, respectively, the spatial and temporal amplitudes of the laser beam. Here again, we assume that this pulse is not modified during its propagation in the nonlinear crystal. After its generation in a very thin nonlinear crystal located at $z=z_{0}$, the THz electric field $E(\Omega, r, z)$ at the transverse plane $z$, is the convolution of the THz field $E\left(\Omega, r, z_{0}\right)$ leaving the crystal with the Fresnel propagator

$$
\frac{\exp \left(i k(\Omega)\left(z-z_{0}\right)\left[1+\frac{r^{2}}{2\left(z-z_{0}\right)^{2}}\right]\right)}{i \lambda\left(z-z_{0}\right)}
$$



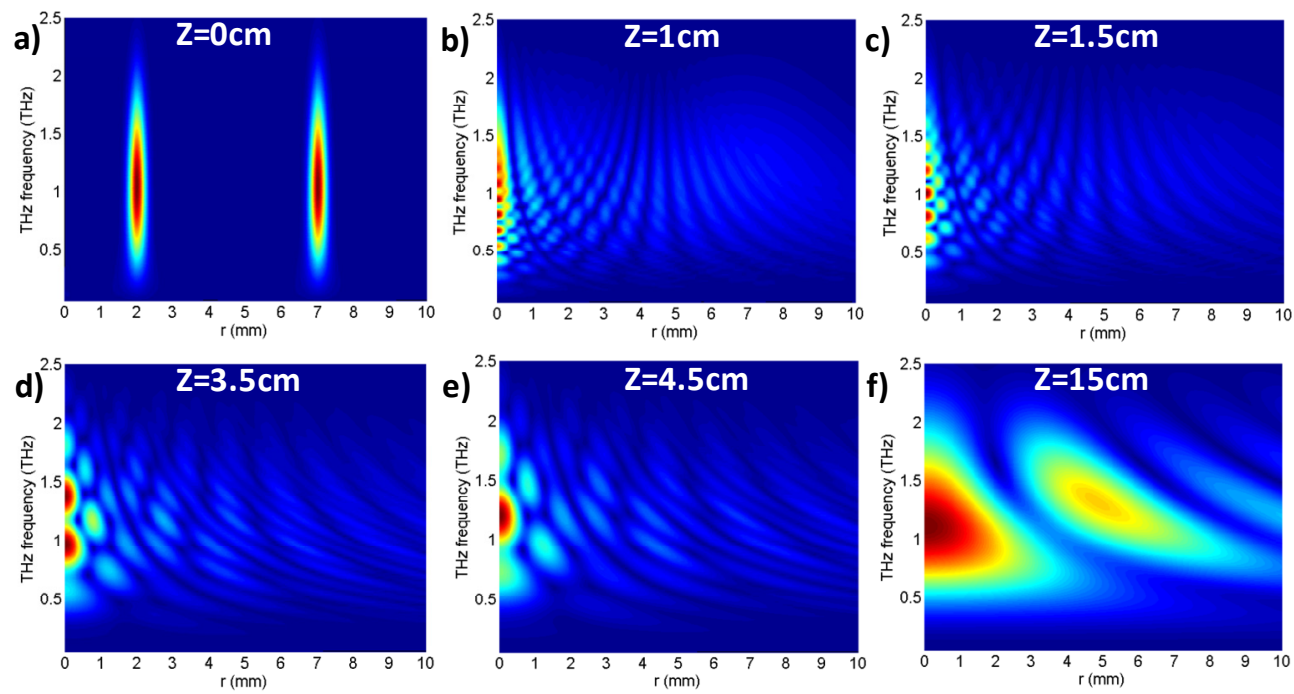

Figure 10. Numerical spatiotemporal behavior of a THz pulse emitted by a ZnTe crystal at different position along the propagation direction. The near I.R. femtosecond pump pattern is represented by two concentric circles that have a radius of $2 \mathrm{~mm}$ and $7 \mathrm{~mm}$ and a thickness of $200 \mu \mathrm{m}$ (taken from [7])

where $\Omega$ and $\lambda$ are respectively the $\mathrm{THz}$ frequency and the associated wavelength. In the spatial frequency domain it reads:

$$
E\left(\Omega, k_{\perp}, z\right)=E\left(\Omega, k_{\perp}, z_{0}\right) \cdot \mathbb{F}^{2}\left(\frac{\exp \left(i k(\Omega)\left(z-z_{0}\right)\left[1+\frac{r^{2}}{2\left(z-z_{0}\right)^{2}}\right]\right)}{i \lambda\left(z-z_{0}\right)}\right) .
$$

The expression of $E\left(\Omega, k_{\perp}, z_{0}\right)$ is given by Eq. (8). If the pump beam has a radial symmetry, the function $G\left(k_{\perp}\right)$ is given by:

$$
G\left(k_{\perp}\right)=2 \pi \int_{0}^{\infty} F(r) J_{0}\left(k_{\perp} r\right) r d r .
$$

It represents the Hankel transform of the pump laser fluence $F(r), J_{0}\left(k_{\perp} r\right)$ being the $0^{\text {th }}$ order Bessel function. The latter equation makes it possible to compute the spatial and spectral profiles of a $\mathrm{THz}$ pulse propagating in a linear medium after its generation in a nonlinear crystal.

To appreciate the possibilities offered by our approach of the near field, the spatiotemporal evolution of the $\mathrm{THz}$ beam generated by a spatially patterned femtosecond laser pulse is shown in Fig. 10. The spatial profile of the pump beam used to generate the THz pulse in a $1 \mathrm{~mm}$-thick ZnTe crystal consists in two concentric circles having a radius of $2 \mathrm{~mm}$ and $7 \mathrm{~mm}$. At the exit of the crystal, the $\mathrm{THz}$ and pump beam pattern matches. However as shown in Fig. 10, along short distances, as the THz pulse propagates in free space most of its energy is confined around the $z$-axis $(r=0)$. In fact, in the $r$ - THz frequency plane, one can observe along $r=0$ the modulation of the $\mathrm{THz}$ spectrum. To account for this 
behavior, we have proposed the following phenomenological interpretation. At the exit of the crystal, each NIR circle simultaneously generates a THz pulse. The THz pulses are emitted as Cherenkov-radiation-like conical beams. This is partly due to the thickness of each circle which is about the $\mathrm{THz}$ central wavelength (or much smaller for lower $\mathrm{THz}$ frequencies) $[4,32]$. Under these conditions, emitted $\mathrm{THz}$ pulses can cross themselves in the intermediate field, generating a $\mathrm{THz}$ pulse train in a given point along the longitudinal axis. As shown in Fig. 10, under favorable geometries, spectral interference are expected along the propagation axis.

\subsection{Comparison between experimental results and theory.}

In order to check the validity of our approach we have used the setup shown in Fig. 11. The pump pulse (about $100 \mu \mathrm{J}, 800 \mathrm{~nm}, 50 \mathrm{fs}$ at $1 \mathrm{kHz}$ ) generates $\mathrm{THz}$ pulses in the first $\mathrm{ZnTe}$ crystal. The THz spectrum generated in the latter crystal is then detected in a second $\mathrm{ZnTe}$ crystal. The measurements are performed along the axis for different distance $d$ between the emitting and detecting ZnTe crystals. Both crystals were $1 \mathrm{~mm}$-thick. The NIR pump and probe beams are separated by mean of a thin undoped silicon wafer. The latter is almost transparent to the THz pulse. As shown in Fig. 11 to pattern the pump beam profile we used a liquid crystal Spatial Light Modulator (SLM). The pump beam reflected by the SLM that carries the spatial phase added by the SLM is focussed by means of a $1 \mathrm{~m}$ focal length lens. At the focal point of the lens, where one records the spatial Fourier transform of the pump beam, we place the first ZnTe crystal. This device makes it possible to impinge patterned pump beam profiles in the ZnTe crystal that generates the THz pulse.

In the first set of experiments, $\mathrm{THz}$ pulse spectra are recorded along the propagation axis, under a two concentric NIR circles cross-section geometry, at three different distances from the generating crystal $(z=1.5 \mathrm{~cm}, 2.5 \mathrm{~cm}$ and $4.5 \mathrm{~cm})$. The two NIR circles have radii of $2 \mathrm{~mm}$ and $4 \mathrm{~mm}$. The result is shown in Fig. 12. Note that the spectrum shape changes completely as the position of the detecting crystal changes, in accordance with the theoretical considerations above. This behavior can be explained by the theoretical model. In fact, the optical path difference between the two THz pulses, simultaneously emitted by the two NIR circles, decreases as the detection distance increases along the $z$-axis. As a consequence the number of interference fringes in the spectrum decreases and the spectral peak moves at the

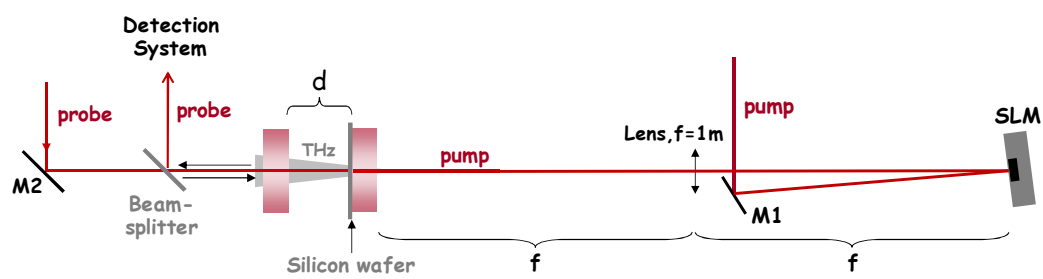

Figure 11. Experimental setup. Two $1 \mathrm{~mm}$-thick ZnTe crystals are used to generate and detect the THz pulse. The pump and probe pulses are delivered by a regenerative amplifier (Coherent Legend) at $800 \mathrm{~nm}$, with $50 \mathrm{fs}$ duration, working at a $1 \mathrm{kHz}$ repetition rate. The pump beam is spatial patterned by a SLM working in reflection. The THz pulse is measured along the propagation direction for different distances $d$ between the generating and detecting crystal. The pump beam is focussed in the generating crystal by means of a $1 \mathrm{~m}$ focal lens. The latter also realizes the spatial Fourier transform (taken from [7]). 
a)

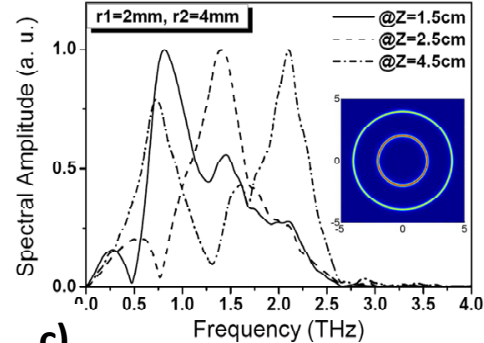

c)
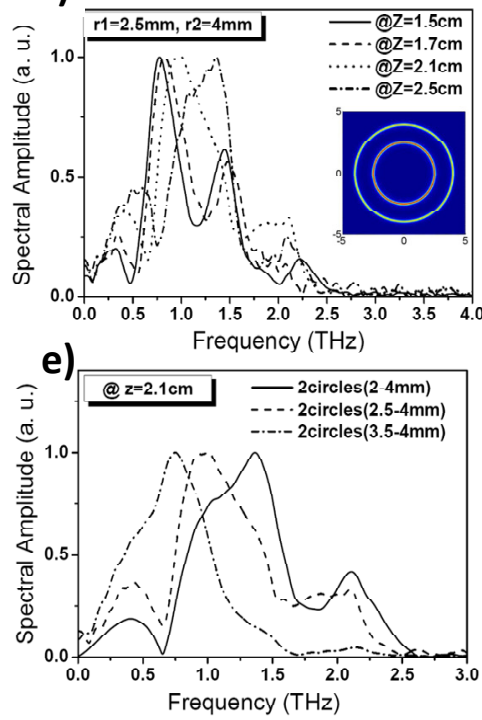

b)

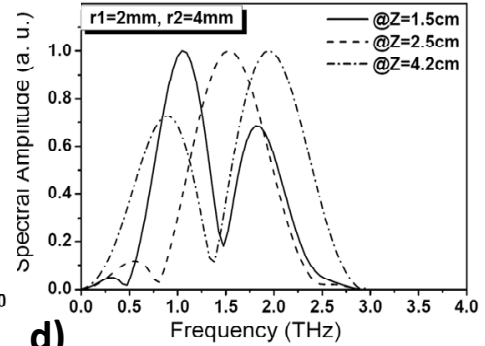

d)
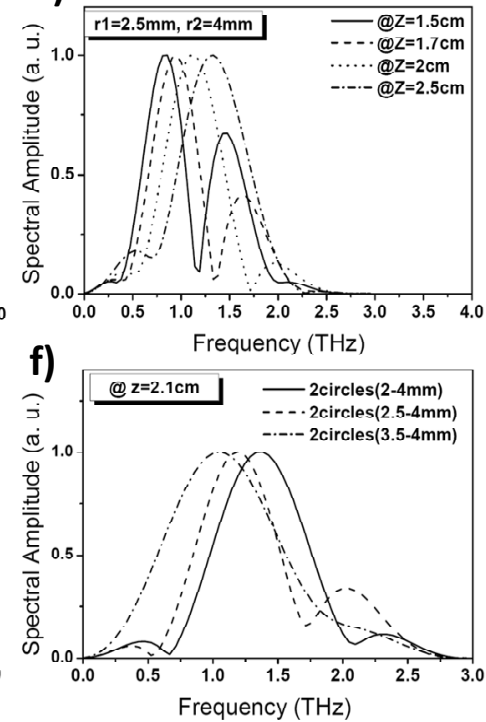

Figure 12. Experimental results (figures $3 a, 3 c, 3 e$ ) and corresponding numerical simulations (figures $3 b$, 3d, 3f) for THz spectra obtained by optical rectification in ZnTe under two concentric circles configurations of the pump laser beam profile. The insets show input beam rings. Each time, spectral amplitudes are normalized (taken from [7]).

same time toward the higher frequencies. A similar result is obtained for two concentric NIR circles with radii of $2.5 \mathrm{~mm}$ and $4 \mathrm{~mm}$, and it is shown in Fig. 12c. Very good agreement is found between experiment (Fig. 12a and c) and numerical simulations (Fig. 12b and d). In the second set of experiments the detecting-ZnTe is placed at a fixed distance of $2.1 \mathrm{~cm}$ from the source-ZnTe. We still use a two concentric circles configuration for the pump geometry fixing the radius of the external circle at $4 \mathrm{~mm}$ but changing the radius of the internal circle from $2 \mathrm{~mm}$ to $3.5 \mathrm{~mm}$. Spectra recorded for three different diameter values are shown in Fig. 12e. Note that the spectral peak moves toward the lower frequencies as the diameter of the internal circle increases. Here again, the numerical simulations (Fig. 12f) are in very good agreement with the experimental results (Fig. 12e).

Another important characteristic of this source is that most of the emitted $\mathrm{THz}$ radiation is spatially confined around the $z$-axis, during propagation, as shown by the numerical 

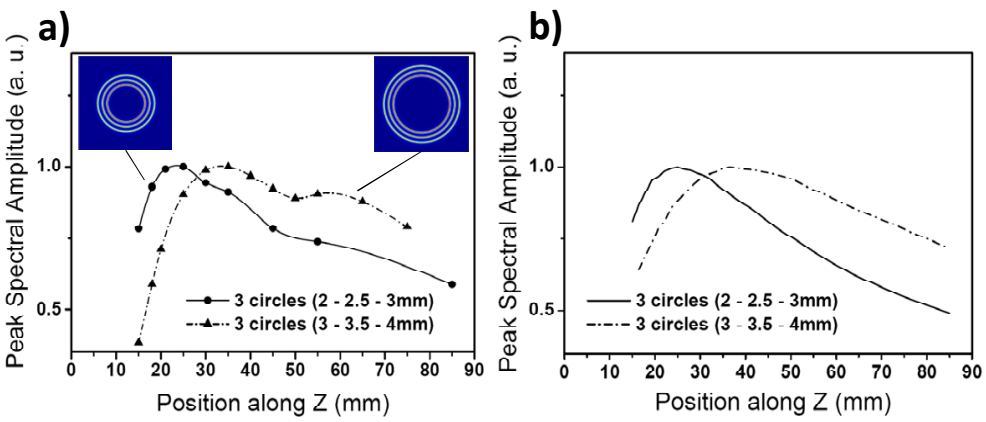

Figure 13. THz peak spectral amplitude against the position of the detecting $\mathrm{ZnTe}$ crystal along $z$. THz radiation shows interesting "self-focusing" properties. The "focal" position changes with the radius values of the circles. Fig. 4a Experiment (the insets show representative ring configuration, lines are guides for eyes); Fig. 4b simulations (taken from [7]).

simulation of Fig. 10. This behavior can be explained by the following simple argumentation. The $z$-axis is the zone of space which minimizes the optical path difference between the pulses coming from each NIR circle. Therefore constructive spatial interference between the spectral components of the $\mathrm{THz}$ pulses generated by each NIR circle occur mainly along the propagation axis. Furthermore, for a given cross-section geometry, the peak spectral amplitude exhibits a maximum value at a given point $z$ (the focal point). The device acts as a tunable chromatic lens. This behavior is shown in the experimental curves of Fig. 13a, obtained using a three concentric circles geometry for the pump fluence. We study two configurations. The first one is represented by three circles with radii of $2 \mathrm{~mm}, 2.5 \mathrm{~mm}$ and $3 \mathrm{~mm}$ respectively (black solid circles); in the second configuration the three circles have radii of $3 \mathrm{~mm}, 3.5 \mathrm{~mm}$ and $4 \mathrm{~mm}$ respectively (black solid triangles). In the first case the peak spectral amplitude exhibits a maximum around $z=20 \mathrm{~mm}$, in the second case the maximum is found around $z=35 \mathrm{~mm}$. Corresponding numerical simulations, in Fig. 13b, are in very good agreement with the experimental results.

\subsection{Generation of spectrally shaped $\mathrm{THz}$ pulses: theoretical approach.}

In the previous sections, we have shown that the use of spatially patterned pump pulses strongly impacts the spectral shape of the near field generated $\mathrm{THz}$ pulses. In fact numerical simulations based on Eq. (34) indicate that very interesting spectrally shaped THz pulses can be achieved under optimized experimental conditions. In Fig. 14 are shown results predicted by the model under two "opposite" geometrical conditions: geometries with few concentric circles widely separated between them (Fig. 14a) and geometries with many close concentric circles (Fig. 14b). In the first case (two circles) the spectrum exhibits a large number of modes, which can be translated along the frequency axis by changing the detection position along $z$ and/or the circle diameters. In the second case (five circles) a very narrow-band THz spectrum can be obtained, and its peak can be translated along the frequency axis by changing the detection position along $z$ and/or the circle diameters. From the experimental point of view, the ZnTe crystal size is a major issue for fine tuning the spectral and spatial shapes of the $\mathrm{THz}$ pulses. In our experiments, the maximum diameter of the circle diameter was limited by the crystal diameter $(10 \mathrm{~mm})$. For instance, the result in Fig. 14a and b can only be achieved 

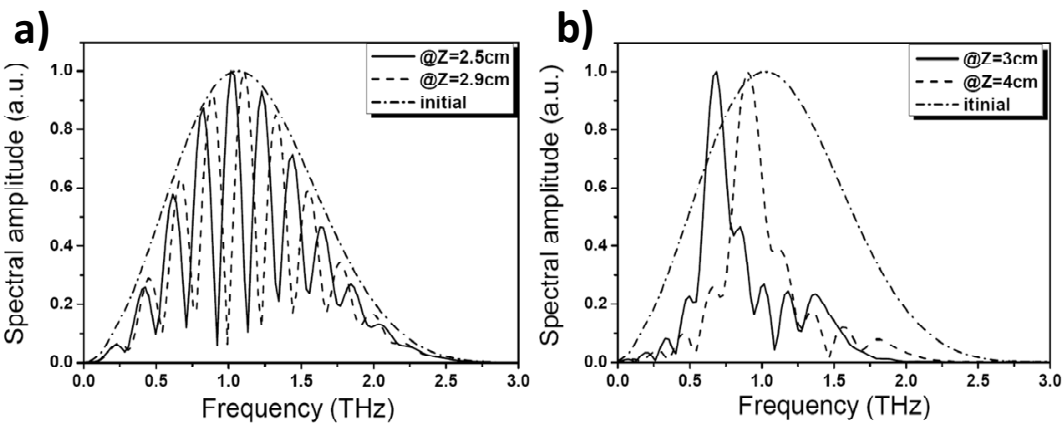

Figure 14. (a) Multi-mode theoretical THz spectra obtained with a two circles configuration (radii of $3 \mathrm{~mm}$ and $9 \mathrm{~mm}$ ), at $2.5 \mathrm{~cm}$ (solid line) and $2.9 \mathrm{~cm}$ (dashed line) from the generating ZnTe. (b) Narrow-band $\mathrm{THz}$ theoretical spectra obtained with a five circles configuration (radii of 11, 12, 13, 14 and $15 \mathrm{~mm}$ ) at $3 \mathrm{~cm}$ (solid line) and $4 \mathrm{~cm}$ (dashed line) from the generating $\mathrm{ZnTe}$. Each time the initial $\mathrm{THz}$ spectrum is shown (dash-dotted line) (taken from [7]).

with $20 \mathrm{~mm}$-large and $30 \mathrm{~mm}$-large ZnTe crystals respectively. Furthermore, due to the initial Gaussian spatial distribution of the pump beam impinging the SLM, NIR circles in the ZnTe crystal have not the same intensity, as a consequence, they generate $\mathrm{THz}$ pulses with unequal amplitude. The good compromises in the present experiment were achieved in Fig. 12 when the circle separation was in between $0.5 \mathrm{~mm}$ and $2 \mathrm{~mm}$, and in between $0.5 \mathrm{~mm}$ and $1 \mathrm{~mm}$ in Fig. 13.

\section{Conclusion}

We have presented two new approaches to synthesize THz pulse sequences and narrow-band tunable THz pulses via optical rectification of shaped NIR pulses in ZnTe. In the first one, the NIR pulses are shaped through a simple sinusoidal and/or triangular spectral phase modulation. Our experimental results show a tunability spanning the bandwidth permitted by the phase matching condition in ZnTe, i.e. ranging from 0.5 to $2.5 \mathrm{THz}$, and the possibility to generate spectra with bandwidth as narrow as $140 \mathrm{GHz}$. These results are in good agreement with a theoretical model taking into account $\mathrm{THz}$ generation by optical rectification, the shape of the optical pulses, dispersion in the $\mathrm{THz}$ range and the electro-optic detection. The second one has been less explored. It relies on the spatial shaping of the NIR pulse beam profile. Here again, we have demonstrated our ability to generate tunable $\mathrm{THz}$ pulses that agreed with our numerical simulations. One should note that this second technique is potentially as versatile as the first one, even if it is not relying on the same kind of pulse shaping method.

One important point is related to the intensity of the $\mathrm{THz}$ pulses we can generate using these techniques. A critical issue is the peak intensity that the used crystal can stand. Indeed, since we are dealing with second order nonlinear effects, one could want to increase as much as possible the peak intensity of the NIR femtosecond pulses. Unfortunately, as one increases the peak intensity new nonlinear phenomena are usually taking place. For example, we have recently shown that increasing the peak intensity of the pump beam in ZnTe induces two-photon absorption that impacts quite strongly the optical rectification efficiency as well as 
the available $\mathrm{THz}$ bandwidth [38]. Hence there is a compromise to find between peak power, crystal thickness, $\mathrm{THz}$ conversion efficiency. Such an effect becomes negligible if one uses for example $\mathrm{LiNbO}_{3}$ crystal, thanks to its higher optical gap. However, this latter crystal has a lower nonlinear $\chi^{(2)}$, a higher intrinsic $\mathrm{THz}$ absorption above $3 \mathrm{THz}$ and a lower $\mathrm{THz}$ spectral acceptance. But the latter limitation can be circumvented using the so-called tilted pulsefront phase matching technique [14], leading to $\mathrm{THz}$ fields with an amplitude up to $400 \mathrm{kV} \cdot \mathrm{cm}^{-1}$ [24]. The combination of tunable $\mathrm{THz}$ pulse generation and nonlinear optics makes it possible to envision new developments in $\mathrm{THz}$ remote sensing, hyperspectral imaging, study and characterization of $\mathrm{THz}$ materials as well as high resolution spectroscopy largely emphasized by recent review articles [13, 14, 34].

\section{Acknowledgments:}

The Conseil Régional d'Aquitaine and the French National Research Agency (ANR 09-BLAN-0212) are acknowledged for financial supports.

\section{Author details}

J. Degert, M. Tondusson, J. Oberlé and É. Freysz

Université de Bordeaux, Laboratoire Ondes et Matiïfre d'Aquitaine, France

S. Vidal

Université de Bordeaux, Laboratoire Ondes et Matiïfire d'Aquitaine, France

Present address: Université de Bordeaux, Centre Lasers Intenses et Applications, France

C. D'Amico

Université de Bordeaux, Laboratoire Ondes et Matiüfre d'Aquitaine, France

Present address: Université Jean Monet, Laboratoire Hubert Curien, France

\section{References}

[1] Ahn, J.; Efimov, A. V.; Averitt, R. D. \& Taylor, A. J. (2003). Terahertz waveform synthesis via optical rectification of shaped ultrafast laser pulses. Opt. Express, Vol. 11, 2486-2496, ISSN 1094-4087

[2] Akhmanov, S. A.; Vysloukh, V. A.; Chirkin, A. S. Optics of Femtosecond Laser Pulses, AIP, ISBN 0883188511, New York

[3] Amand, T.; Blanchet, V.; Girard, B. \& Marie, X. (2005). Coherent Control in Atoms, Molecules and Solids, In: Femtosecond Laser Pulses, C. Rullière, (Ed.), 333-394, Springer-Verlag, ISBN 1441918507, New-York

[4] Auston, D. H. ; Cheung, K. P.; Valdmanis, J. A. \& D. A. Kleinman, D. A. (1984). Cherenkov Radiation from Femtosecond Optical Pulses in Electro-Optic Media. Phys. Rev. Lett., Vol. 53, 1555-1558, ISSN 0031-9007

[5] Brener, I.; Planken, P. C. M.; Nuss, M. C.; Pfeiffer, L.; Leaird, D. E. \& Weiner, A. M. (1993). Repetitive excitation of charge oscillations in semiconductor heterostructures. Appl. Phys. Lett., Vol. 63, 2213-2215, ISSN 0003-6951 
[6] Caumes, J.-P.; Videau, L.; Rouyer, C. \& Freysz, E. (2002). Kerr-Like Nonlinearity Induced via Terahertz Generation and the Electro-Optical Effect in Zinc Blende Crystals. Phys. Rev. Lett., Vol. 89, 047401, ISSN 0031-9007

[7] D'Amico, C.; Tondusson, M.; Degert, J. \& Freysz, E. (2009). Tuning and focusing THz pulses by shaping the pump laser beam profile in a nonlinear crystal. Opt. Express, Vol. 17, 592-597, ISSN 1094-4087

[8] Danielson, J. R.; Jameson, A. D.; Tomaino, J. L.; Hui, H.; Wetzel, J. D.; Lee, Y.-S. \& Vodopyanov, K. L. (2008). Intense narrow band terahertz generation via type-II difference-frequency generation in ZnTe using chirped optical pulses. J. Appl. Phys., Vol. 104, 03311, ISSN 0021-8979

[9] Feurer, T.; Vaughan, J. C. \& Nelson, K. A. (2003). Spatiotemporal Coherent Control of Lattice Vibrational Waves. Science, Vol. 299, 374-377, ISSN 1095-9203

[10] Feurer, T.; Vaughan, J. C.; Hornung, T. \& Nelson, K. A. (2004). Typesetting of terahertz waveforms. Opt. Lett., Vol. 29, 1802-1804, ISSN 0146-9592

[11] Gallot, G. \& Grischkowsky, D. (1999). Electro-optic detection of terahertz radiation. J. Opt. Soc. Am. B, Vol. 16, 1204-1212, ISSN 0740-3224

[12] Gallot, G.; Zhang, J.; McGowan, R. W.; Jeon, T. \& Grischkowsky, D. (1999). Measurements of the $\mathrm{THz}$ absorption and dispersion of ZnTe and their relevance to the electro-optic detection of THz radiation. Appl. Phys. Lett., Vol. 74, 3450-3452, ISSN 0003-6951

[13] Hebling, J. ; Yeh, K.-L.; Hoffmann, M. C. \& Nelson, K. A. (2008). High Power THz Generation, THz Nonlinear Optics, and THz Nonlinear Spectroscopy. IEEE J. Selected Topics in Quantum Electronics, Vol. 14, 345-353, ISSN 1077-260X

[14] Hoffmann, M.C. \& Fülöp, J.A. (2001). Intense ultrashort terahertz pulses: Generation and applications. J. Phys. D: Appl. Phys., Vol. 44, 083001, ISSN 0022-3727

[15] Huggard, P. G.; Cluff, J. A.; Shaw, C. J.; Andrews, S. R.; Linfield, E. H. \& Ritchie, D. A. (1997). Coherent control of cyclotron emission from a semiconductor using sub-picosecond electric field transients. Appl. Phys. Lett., Vol. 71, 2647-2649, ISSN 0003-6951

[16] Hurlbut, W. C.; Norton, B. J.; Amer, N. \& Lee, Y.-S. (2006). Manipulation of terahertz waveforms in nonlinear optical crystals by shaped optical pulses. J. Opt. Soc. B, Vol. 23, 90-93, ISSN 0740-3224

[17] Kohli, K. K.; Vaupel, A.; Chatterjee, S. \& Rühle, W. W. (2009). Adaptive shaping of THz-pulses generated in $\langle 100\rangle$ ZnTe crystals. J. Opt. Soc. B, Vol. 26, 74-78, ISSN 0740-3224

[18] Lee, Y.-S.; Meade, T.; Norris, T. B. \& Galvanauskas, A. (2001). Tunable narrow-band terahertz generation from periodically poled lithium niobate. Appl. Phys. Lett., Vol. 78, 3583-3585, ISSN 0003-6951

[19] Lee, Y.-S.; Amer, N. \& Hurlbut, W. C. (2003). Terahertz pulse shaping via optical rectification in poled lithium niobate. Appl. Phys. Lett., Vol. 82, 170-172, ISSN 0003-6951

[20] Lee, Y.-S. (2009). Principles of Terahertz Science and Technology, Springer-Verlag, ISBN 038709539X, New-York.

[21] Liu, Y.; Park, S.-G. \& Weiner, A. M. (1996). Terahertz Waveform Synthesis via Optical Pulse Shaping. IEEE J. Sel. Top. Quantum Electron., Vol. 2, 709-719, ISSN 1077-260X

[22] Monmayrant, A. \& Chatel, B. (2004). New phase and amplitude high resolution pulse shaper. Rev. Sci. Instrum., Vol. 75, 2668-2671, ISSN 0034-6748 
[23] Monmayrant, A.; Weber, S. \& Chatel, B. (2010). A newcomer's guide to ultrashort pulse shaping and characterization. J. Phys. B: At. Mol. Opt. Phys., Vol. 43, 103001, ISSN 0953-4075

[24] Nagai, M.; Matsubara, E. \& Ashida, M. (2012). High-efficiency terahertz pulse generation via optical rectification by suppressing stimulated Raman scattering process. Opt. Express, Vol. 20, 6509-6514, ISSN 1094-4087

[25] Nahata, A.; Weling, A. S. \& Heinz, T. F. (1996). A wideband coherent terahertz spectroscopy system using optical rectification and electro-optic sampling. Appl. Phys. Lett., Vol. 69, 2321-2323, ISSN 0003-6951

[26] Planken, P. C. M.; Brener, I.; Nuss, M. C.; Luo, M. S. C. \& Chuang, S. L. (1993). Coherent control of terahertz charge oscillations in a coupled quantum well using phase-locked optical pulses. Phys. Rev. B, Vol. 48, 4903-4906, ISSN 1098-0121

[27] Qi, T.; Shin, Y.-H.; Yeh, K.-L.; Nelson, K. A. \& Rappe, A. M. (2009). Collective Coherent Control: Synchronization of Polarization in Ferroelectric $\mathrm{PbTiO}_{3}$ by Shaped $\mathrm{THz}$ Fields. Phys. Rev. Lett., Vol. 102, 247603, ISSN 0031-9007

[28] Reimann, K. (2007). Table-top sources of ultrashort THz pulses. Rep. Prog. Phys., Vol. 70, 1597-1632, ISSN 0034-4885.

[29] Renard, M.; Chaux, R.; Lavorel, B. \& Faucher, O. (2004). Pulse trains produced by phase-modulation of ultrashort optical pulses: tailoring and characterization. Opt. Express, Vol. 12, 473-482, ISSN 1094-4087

[30] Sohn, J. Y.; Ahn, Y. H.; Park, D. J.; Oh, E. \& Kim, D. S. (2002). Tunable terahertz generation using femtosecond pulse shaping. Appl. Phys. Lett., Vol. 81, 13-15, ISSN 0003-6951

[31] Stepanov, A. G.; Hebling, J. \& Kuhl, J. (2004). Generation, tuning, and shaping of narrow-band, picosecond $\mathrm{THz}$ pulses by two-beam excitation. Opt. Express, Vol. 12, 4650-4658, ISSN 1094-4087

[32] Stepanov, A. G.; Hebling, J. \& Kuhl, J. (2005). THz generation via optical rectification with ultrashort laser pulse focused to a line. Appl. Phys. B, Vol. 81, 23-26, ISSN 0946-2171

[33] Stobrawa, G.; Hacker, M.; Feurer, T.; Zeidler, D.; Motzkus, M. \& Reichel, F. (2001). A new high-resolution femtosecond pulse shaper. Appl. Phys. B, Vol. 72, 627-630, ISSN 0946-2171

[34] Tanaka, M.; Hirori, H. \& Nagai, M. (2011). THz Nonlinear Spectroscopy of Solids. IEEE Transactions on Terahertz Science and Technology, Vol. 1, 301-312, ISSN 2156-342X

[35] Vaughan, J. C.; Feurer, T.; Stone, K. W. \& Nelson, K. A. (2006). Analysis of replica pulses in femtosecond pulse shaping with pixelated devices. Opt. Express, Vol. 14, 1314-1328, ISSN 1094-4087

[36] Vaughan, J. C.; Hornung, T.; Feurer, T. \& Nelson, K. A. (2005). Diffraction-based femtosecond pulse shaping with a two-dimensional spatial light modulator. Opt. Lett., Vol. 30, 323-325, ISSN 0146-9592

[37] Vidal, S.; Degert, J.; Oberlï $£_{i}$, J. \& Freysz, E. (2010). Femtosecond optical pulse shaping for tunable terahertz pulses generation. J. Opt. Soc. Am. B., Vol. 27, 1044-1050, ISSN 0740-3224

[38] Vidal, S.; Degert, J.; Tondusson, M.; Oberli $£_{i}$, J. \& Freysz, E. (2011). Impact of dispersion, free carriers and two-photon absorption on the generation of intense THz pulses in ZnTe crystals. Appl. Phys. Lett., Vol. 98, 191103, ISSN 0003-6951 
[39] Wahlstrand, J. K. \& Merlin, R. (2003). Cherenkov radiation emitted by ultrafast laser pulses and the generation of coherent polaritons. Phys. Rev. B, Vol. 68, 054031, ISSN 1098-0121

[40] Ward, D. W.; Beers, J. D.; Feurer, T.; Statz, E. R.; Stoyanov, N. S. \& Nelson, K. A. (2004). Coherent control of phonon-polaritons in a terahertz resonator fabricated with femtosecond laser machining. Opt. Lett., Vol. 29, 2671-2673, ISSN 0146-9592

[41] Weiner, A. M.; Leaird, D. E.; Wiederrecht, G. P. \& Nelson, K. A. (1990). Femtosecond Pulse Sequences Used for Optical Manipulation of Molecular Motion. Science, Vol. 247, 1317-1319, ISSN 1095-9203

[42] Weiner, A. M. (2000). Femtosecond pulse shaping using spatial light modulators. Rev. Sci. Instrum., Vol. 71, 1929-1960, ISSN 0034-6748

[43] Yamaguchi, M. \& Das, J. (2009). Terahertz wave generation in nitrogen gas using shaped optical pulses. J. Opt. Soc. B, Vol. 26, 90-94, ISSN 0740-3224

[44] Yano, R.; Nakagawa, K. \& Shinojima, H. (2009). Phase and Amplitude Control of Free Induction Decay Emitted from Water Vapor at $0.55 \mathrm{THz}$ Transition. Jpn. J. Appl. Phys., Vol. 48, 022401, ISSN 0021-4922 